\title{
Lysine methylation shields an intracellular pathogen from ubiquitylation
}

\author{
Patrik Engström ${ }^{*}$, Thomas P. Burke ${ }^{1}$, Anthony T. Iavarone ${ }^{2}$, Matthew D. Welch ${ }^{*}$ \\ ${ }^{1}$ Department of Molecular and Cell Biology, University of California, Berkeley, CA 94720, USA. \\ ${ }^{2}$ QB3/Chemistry Mass Spectrometry Facility, University of California, Berkeley, CA 94720, USA. \\ *Correspondence to: pengstrom@berkeley.edu (P.E.), welch@berkeley.edu (M.D.W).
}




\section{Abstract}

Many intracellular pathogens avoid detection by their host cells. However, it remains

3 unknown how they avoid being tagged by ubiquitin, an initial step leading to anti-microbial

4 autophagy. Here, we show that the intracellular bacterial pathogen Rickettsia parkeri uses

5 two protein-lysine methyltransferases (PKMTs) to modify outer membrane proteins (OMPs) and

6 prevent their ubiquitylation. Mutants deficient in the PKMTs were avirulent in mice and failed to

7 grow in macrophages due to ubiquitylation and autophagy. Analysis of the lysine-methylome

8 revealed that PKMTs modify a subset of OMPs by methylation at the same sites that are recognized

9 by host ubiquitin. These findings show that lysine methylation is an essential determinant of

10 rickettsial pathogenesis that shields bacterial proteins from ubiquitylation to evade autophagic

11 targeting. 
Main

Intracellular pathogens generally evade the host immune surveillance machinery. This

14 includes avoidance of surface targeting by the host ubiquitylation machinery and subsequent

15 formation of a polyubiquitin coat, a first step in cell-autonomous immunity (1-5). The ubiquitin

16 coat recruits autophagy receptors that engage with the autophagy machinery to target cytosol-

17 exposed microbes for destruction $(1,4-8)$. Bacterial outer membrane proteins (OMPs) are targets

18 for the host ubiquitylation machinery $(9,10)$, and the tick-borne obligate intracellular pathogen

19 Rickettsia parkeri requires the abundant surface protein OmpB to protect OMPs from

20 ubiquitylation (11). However, the detailed mechanisms that $R$. parkeri and other pathogens use to

21 block lysine ubiquitylation of surface proteins, including OMPs, are unknown. We hypothesized

22 that cell surface structures or modifications could provide protection at the molecular level. One

23 such modification is lysine methylation, which is widespread in all domains of life. This

24 modification involves the transfer of one, two, or three methyl groups to the amino group of lysine

25 side chains, the same amino group that can also be modified by ubiquitin (12). Whether lysine

26 methylation of bacterial surfaces prevents host detection and promotes intracellular survival has

27 not been explored.

To identify bacteria-derived surface modifications that protect against ubiquitin coating,

29 we screened a library of $R$. parkeri transposon mutants (13) (Table S1) for increased

30 polyubiquitylation ( $\mathrm{pUb}$ ) relative to wild-type (WT) in Vero cells (an epithelial cell line commonly

31 used to propagate and study intracellular pathogens) by immunofluorescence microscopy (Fig.

32 1A). We subsequently analyzed individual mutants and identified 4 that were ubiquitylated, similar

33 to $\mathrm{ompB}$ mutant bacteria (11) (Fig. 1B and D). Importantly, these 4 strains expressed OmpB (Fig.

34 S1). Two of the strains had insertions in the protein-lysine methyltransferase genes $p k m t 1$ and 
pkmt2, which are located in two distinct chromosomal regions. The remaining two strains had insertions in the wecA and $r m l D$ genes, which are required for the biosynthesis of O-antigen (Fig. S2), a common surface molecule in Gram-negative bacteria. The O-antigen also protects Francisella tularensis from ubiquitylation (5), suggesting it performs a conserved function in this process. As a control, we analyzed a strain with a mutation in the $\operatorname{mrdA}$ gene, which is required

40 for peptidoglycan biosynthesis and cell shape in other bacteria (14). This mutant strain had altered

41 shape but was not polyubiquitylated (Fig. 1B and D), suggesting that not all bacterial cell envelope

42 structures are required to avoid ubiquitylation. In addition, we further quantified the pUb levels

43 and observed that the pkmt1::tn mutant, followed by the ompB and $p k m t 2::$ tn mutants, had the

44 highest levels (Fig. 1E). These data indicated that OmpB, PKMTs, and the O-antigen protect $R$.

45 parkeri from ubiquitylation.

The O-antigen was previously shown to be required for rickettsial pathogenesis (15), and

47 OmpB was previously found to be required for $R$. parkeri to cause lethal disease in Ifnar ${ }^{-/}$Ifngr $^{-/-}$ mice lacking the type I interferon receptor (IFNAR) and IFN-g receptor (IFNGR) (16). We therefore examined whether PKMT1 or PKMT2 are important for causing disease in vivo. We observed that Ifnar--Ifngr-/- mice succumbed to infection with WT but not to $p k m t 1::$ tn or pkmt2::tn

51 bacteria (Fig. 1F). Mice infected with the $p k m t 1:$ tn mutant showed no signs of disease, whereas

52 mice infected with pkmt2::tn showed a transient loss in body weight (Fig. S3). This indicates that

53 PKMT1 and PKMT2 are determinants of $R$. parkeri pathogenesis.

Because PKMT1 or PKMT2 had previously been shown to methylate OmpB in vitro (17-

5520 ), we next examined whether methylation protects $\mathrm{OmpB}$, or another abundant outer membrane

56 protein OmpA (21), from ubiquitylation. First, Vero cells overexpressing 6xHis-tagged ubiquitin

57 were infected with WT and the ompB, pkmt1::tn, and pkmt2::tn strains. 6xHis-tagged ubiquitin 
58 was recruited to the surface of all of the mutants, but not WT bacteria, as observed by

59 immunofluorescence microscopy (Fig. 2A). Then, 6xHis-ubiquitylated proteins were affinity-

60 purified from infected cells, and OmpA and OmpB were detected by western blotting. OmpA was

61 shifted towards higher molecular weights in cells infected with mutant, but not WT bacteria,

62 indicating OmpA is ubiquitylated (Fig. 2B). Similarly, in comparison with WT bacteria, OmpB

63 was also shifted towards higher as well as lower molecular weights in cells infected with the

$64 p k m t 1::$ tn and $p k m t 2::$ tn mutants, suggesting that OmpB is ubiquitylated (Fig. 2B). To confirm that

65 methylation protects $\mathrm{OmpB}$ and OmpA from ubiquitylation on the bacterial surface, we performed

66 pUb-enrichments of surface fractions from purified bacteria followed by western blotting. This

67 revealed that both $\mathrm{OmpB}$ and OmpA shifted towards higher molecular weights in the

68 methyltransferase mutants but not in WT bacteria (Fig. 2C). Thus, methylation is critical to protect

69 OMPs from ubiquitylation on the bacterial surface.

70 Based on our observation that methylation protects both OmpA and OmpB from

71 ubiquitylation, we set out to determine how frequently, and to what extent, lysines of $R$. parkeri

72 OMPs are methylated. Peptides with methylated lysines from whole WT bacteria were quantified

73 using label-free liquid chromatography-mass spectrometry (LC-MS). We then analyzed lysine

74 methylation frequency in abundant OMPs as well as other abundant $R$. parkeri proteins (Table

75 S2). This analysis revealed that $R$. parkeri OmpB, OmpA, and surface cell antigen 2 (Sca2)

76 proteins had the highest abundance of methylated peptides. Lysine methylation was also detected

77 in the outer-membrane assembly protein BamA and in a predicted outer membrane protein porin

78 (WP_014410329.1; from here on referred to as OMP-porin) (Fig. 3A and Table S3). We next

79 mapped both methylated and unmethylated lysines on the above-mentioned OMPs and found that

80 more than $50 \%$ of lysines detected from OmpB and OmpA were methylated, and a significant 
81 fraction of lysines were also methylated in Sca2 (31\%), OMP-porin (30\%), and BamA (27\%) (Fig.

$82 \quad 3 B$ and Fig. S4). Thus, in R. parkeri, lysine methylation of OMPs is common.

To identify OMPs that are methylated by PKMT1 and PKMT2 during infection, we

84 compared lysine methylation frequencies in WT with those of $p k m t 1::$ tn or $p k m t 2::$ tn mutant

85 bacteria using LC-MS. We found that monomethylation of OmpB, OmpA, the predicted OMP-

86 porin, and another surface cell antigen protein Sca1 were reduced in pkmt1::tn compared to WT

87 bacteria (Fig. 3C and Fig. S5). Dimethylation of rickettsial surface proteins was not reduced in the

88 mutants (Fig. S6). Although trimethylation was rare and therefore difficult to analyze at the

89 individual protein level (Fig. S6), OmpB had reduced trimethylation levels in both

90 methyltransferase mutants (Fig. 3C). Notably, the frequency of unmethylated lysines in OmpB

91 was specifically increased in pkmt1::tn bacteria (Fig. 3C and Fig. S5). Lysine methylation of five

92 other surface proteins (Sca1, Sca2, BamA, LomR, and Pal-lipoprotein), and 21 of 23 abundant

93 proteins with different predicted subcellular distributions, was not affected by mutations in the

94 pkmt1 or pkmt2 genes (Fig. S5). These data indicate that the PKMTs are required for methylation

95 of a specific subset of OMPs including OmpB.

96 To determine which OmpB residues are modified by PKMT1 and PKMT2 during $R$.

97 parkeri infection, we analyzed the methylation frequency of individual lysines in the mutants

98 compared to WT using LC-MS. We observed reduced monomethylation frequencies of OmpB

$99 \mathrm{~K} 418, \mathrm{~K} 623, \mathrm{~K} 634, \mathrm{~K} 902, \mathrm{~K} 1061, \mathrm{~K} 1294$, and K1323 in pkmt1::tn bacteria compared with WT,

100 and reduced trimethylation frequencies on K1061 and K388 in the pkmt2::tn strain (Fig. 3D).

101 These data indicate that several lysines in $R$. parkeri OmpB are methylated by PKMT1 and

102 PKMT2 during infection. Although these results were consistent with previous biochemical results

103 indicating that PKMT1 monomethylates and PKMT2 trimethylates OmpB's lysines $(17,19)$, we 
104 found that total OmpB-methylation is unaffected in the pkmt2::tn mutant. This suggests that 105 PKMT1 is a primary methyltransferase for OmpB and that it can compensate, at least partly, for a 106 deficiency in PKMT2. residues from ubiquitylation, we performed pUb-enrichments of bacterial surface fractions

109 followed by LC-MS to quantify lysines with diglycine (diGly) remnants, a signature for ubiquitin

110 after trypsin digestion. A prediction of this hypothesis is that individual lysines that are heavily 111 methylated in OmpB of Rickettsia species (17), including WT R. parkeri (Fig. 3D), are targets for 112 ubiquitylation in pkmt1::tn bacteria. In support of this hypothesis, OmpB K634 and K623 in 113 pkmt1::tn exhibited 7 to 10,000-fold increased ubiquitylation compared with WT bacteria (Fig. 114 3E, F and Fig. S7; Table S4). Furthermore, we observed a 13-fold increase in ubiquitylation of 115 the OMP-porin in pkmt1::tn bacteria (Table S4), indicating that methylation also protects 116 additional OMPs. However, in the pkmt2::tn mutant, differential OMP-ubiquitylation was below 117 detection limits (Fig. 3E, F and Table S4). Together, these data indicate that methylation by 118 PKMT1 camouflages lysines in OMPs from ubiquitylation.

119 Because polyubiquitylation promotes recruitment of the autophagy receptors 120 p62/SQSTM1 and NDP52 (7, 8), we hypothesized that lysine methylation shields OMPs from 121 ubiquitylation to block recruitment of these proteins. Consistent with this hypothesis, we observed 122 that the majority of $p k m t 1:: \operatorname{tn}, p k m t 2::$ tn, as well as $o m p B$ mutant bacteria, co-localized with p62 123 and NDP52 by immunofluorescence microscopy (Fig. S8). These data demonstrate that PKMTs 124 protect $R$. parkeri from autophagy recognition. Many pathogenic bacteria including $R$. parkeri grow in immune cells such as macrophages 126 (11), despite the fact that microbial detection in such cells triggers anti-bacterial pathways. We 
127 therefore investigated whether PKMT1 or PKMT2 were required for evading autophagy targeting

128 and bacterial growth in cultured bone marrow-derived macrophages (BMDMs), as was observed

129 for OmpB (11). BMDMs were generated from control mice and mice lacking the gene encoding

130 for autophagy related 5 (ATG5), a protein required for optimal membrane envelopment around

131 pathogens targeted by autophagy, and for their subsequent destruction $(6)$. We observed that

132 pkmt $1:$ tn mutant bacteria were unable to grow in control BMDMs $\left(\operatorname{Atg} 5^{\text {floxflox }}\right)$, and that growth

133 was rescued in $\operatorname{Atg} 5$-deficient BMDMs $\left(\operatorname{Atg}^{-/-}\right)$. Further, $>91 \%$ of $p k m t 1:$ :tn bacteria were labeled

134 with both pUb and p62 in Atg5-deficient BMDMs (Fig. 4A, B, C and D), suggesting that ubiquitin-

135 tagged bacteria were not restricted when the autophagy cascade was prevented. In contrast, the

136 pkmt2::tn mutant did not have a major growth defect compared with WT bacteria and 50\% of the

137 bacteria were labeled with p62, irrespective of host genotype (Fig. 4A, B, C and D), consistent

138 with less pronounced ubiquitylation phenotypes compared to pkmt1::tn bacteria. Collectively,

139 these data indicate that methylation is required for $R$. parkeri growth in macrophages by avoiding

140 autophagic targeting.

Our work reveals a detailed molecular mechanism that camouflages bacterial surface

142 proteins from host detection. In particular, we found that lysine methylation was essential for

143 blocking ubiquitylation, a first step in cell-autonomous immunity (1-4). This highlights an intricate

144 evolutionary arms race between pathogens and hosts and reveals a strategy that pathogens can

145 adapt to counteract host responses. The lysine methyltransferases PKMT1 and PKMT2 are

146 conserved between rickettsial species (Fig. S9 and Fig. S10) and contain a core Rossmann fold

147 found in the broader superfamily of class I methyltransferases that exist in diverse organisms (12,

148 18). Thus, we propose that lysine methylation, and potentially other lysine modifications, could be

149 used by pathogens, symbionts, and perhaps even in eukaryotic organelles, to prevent undesirable 
bioRxiv preprint doi: https://doi.org/10.1101/2020.1120.392290; this version posted December 10,2020 . The copyright holder for this preprint

(which was not certified by peer review) is the author/funder, who has granted bioRxiv a license to display the preprint in perpetuity. It is made available under aCC-BY-NC-ND 4.0 International license.

150 surface ubiquitylation and downstream consequences including elimination by autophagy. Further

151 study of microbial surface modifications will continue to enhance our understanding of the

152 pathogen-host interface and could ultimately lead to new therapeutic targets for treating human

153 diseases including those caused by infectious agents. 


\section{References and Notes}

155 1. F. Randow, R. J. Youle, Self and nonself: how autophagy targets mitochondria and bacteria. Cell Host Microbe 15, 403-411 (2014).

2. A. J. Perrin, X. Jiang, C. L. Birmingham, N. S. So, J. H. Brumell, Recognition of bacteria in the cytosol of Mammalian cells by the ubiquitin system. Curr Biol 14, 806-811 (2004).

3. S. J. L. van Wijk et al., Linear ubiquitination of cytosolic Salmonella Typhimurium activates NF-kappaB and restricts bacterial proliferation. Nat Microbiol 2, 17066 (2017).

4. J. Noad et al., LUBAC-synthesized linear ubiquitin chains restrict cytosol-invading bacteria by activating autophagy and NF-kappaB. Nat Microbiol 2, 17063 (2017).

5. E. D. Case et al., The Francisella O-antigen mediates survival in the macrophage cytosol via autophagy avoidance. Cell Microbiol 16, 862-877 (2014).

6. J. Huang, J. H. Brumell, Bacteria-autophagy interplay: a battle for survival. Nat Rev Microbiol 12, 101-114 (2014).

7. Y. T. Zheng et al., The adaptor protein p62/SQSTM1 targets invading bacteria to the autophagy pathway. J Immunol 183, 5909-5916 (2009).

8. T. L. Thurston, G. Ryzhakov, S. Bloor, N. von Muhlinen, F. Randow, The TBK1 adaptor and autophagy receptor NDP52 restricts the proliferation of ubiquitin-coated bacteria. Nat Immunol 10, 1215-1221 (2009).

9. E. Fiskin, T. Bionda, I. Dikic, C. Behrends, Global analysis of host and bacterial ubiquitinome in response to Salmonella Typhimurium infection. Mol Cell 62, 967-981 (2016).

10. M. Polajnar, M. S. Dietz, M. Heilemann, C. Behrends, Expanding the host cell ubiquitylation machinery targeting cytosolic Salmonella. EMBO Rep 18, 1572-1585 (2017).

11. P. Engström et al., Evasion of autophagy mediated by Rickettsia surface protein OmpB is critical for virulence. Nat Microbiol 4, 2538-2551 (2019).

12. S. Lanouette, V. Mongeon, D. Figeys, J. F. Couture, The functional diversity of protein lysine methylation. Mol Syst Biol 10, 724 (2014).

13. R. L. Lamason, N. M. Kafai, M. D. Welch, A streamlined method for transposon mutagenesis of Rickettsia parkeri yields numerous mutations that impact infection. PLoS One 13, e0197012 (2018).

14. B. G. Spratt, A. B. Pardee, Penicillin-binding proteins and cell shape in E. coli. Nature 254, 516-517 (1975).

15. H. K. Kim, R. Premaratna, D. M. Missiakas, O. Schneewind, Rickettsia conorii O antigen is the target of bactericidal Weil-Felix antibodies. Proc Natl Acad Sci U S A 116, 1965919664 (2019).

16. T. P. Burke et al., Inflammasome-mediated antagonism of type I interferon enhances Rickettsia pathogenesis. Nat Microbiol 5, 688-696 (2020).

17. D. C. H. Yang, A. H. Abeykoon, B. E. Choi, W. M. Ching, P. B. Chock, Outer membrane protein OmpB methylation may mediate bacterial virulence. Trends Biochem Sci 42, 936945 (2017).

18. A. H. Abeykoon et al., Structural insights into substrate recognition and catalysis in outer membrane protein B (OmpB) by protein-lysine methyltransferases from Rickettsia. J Biol Chem 291, 19962-19974 (2016).

19. A. Abeykoon et al., Multimethylation of Rickettsia OmpB catalyzed by lysine methyltransferases. J Biol Chem 289, 7691-7701 (2014). 
20. A. H. Abeykoon et al., Two protein lysine methyltransferases methylate outer membrane protein B from Rickettsia. J Bacteriol 194, 6410-6418 (2012). 6, (2015).

\section{N. F. Noriea, T. R. Clark, T. Hackstadt, Targeted knockout of the Rickettsia rickettsii
OmpA surface antigen does not diminish virulence in a mammalian model system. mBio \\ 21. N. F. Noriea, T. R. Clark, T. Hackstadt, Targeted knockout of the Rickettsia rickettsii
OmpA surface antigen does not diminish virulence in a mammalian model system. mBio}

22. R. L. Lamason et al., Rickettsia Sca4 Reduces Vinculin-Mediated Intercellular Tension to Promote Spread. Cell 167, 670-683 e610 (2016).

23. U. Distler et al., Drift time-specific collision energies enable deep-coverage dataindependent acquisition proteomics. Nat Methods 11, 167-170 (2014).

24. R. S. Plumb et al., UPLC/MS(E); a new approach for generating molecular fragment information for biomarker structure elucidation. Rapid Commun Mass Spectrom 20, 19891994 (2006).

25. P. V. Shliaha, N. J. Bond, L. Gatto, K. S. Lilley, Effects of traveling wave ion mobility separation on data independent acquisition in proteomics studies. J Proteome Res 12, 2323 2339 (2013).

26. S. Nahnsen, C. Bielow, K. Reinert, O. Kohlbacher, Tools for label-free peptide quantification. Mol Cell Proteomics 12, 549-556 (2013).

27. K. A. Neilson et al., Less label, more free: approaches in label-free quantitative mass spectrometry. Proteomics 11, 535-553 (2011).

28. C. I. Carlström et al., (Per)chlorate-reducing bacteria can utilize aerobic and anaerobic pathways of aromatic degradation with (per)chlorate as an electron acceptor. mBio $\mathbf{6}$, (2015).

22. R. L. Lamason et al., Rickettsia Sca4 Reduces Vinculin-Mediated Intercellular Tension to Promote Spread. Cell 167, 670-683 e610 (2016).

23. U. Distler et al., Drift time-specific collision energies enable deep-coverage dataindependent acquisition proteomics. Nat Methods 11, 167-170 (2014).

24. R. S. Plumb et al., UPLC/MS(E); a new approach for generating molecular fragment information for biomarker structure elucidation. Rapid Commun Mass Spectrom 20, 19891994 (2006).

25. P. V. Shliaha, N. J. Bond, L. Gatto, K. S. Lilley, Effects of traveling wave ion mobility separation on data independent acquisition in proteomics studies. J Proteome Res 12, 23232339 (2013).

26. S. Nahnsen, C. Bielow, K. Reinert, O. Kohlbacher, Tools for label-free peptide quantification. Mol Cell Proteomics 12, 549-556 (2013).

27. K. A. Neilson et al., Less label, more free: approaches in label-free quantitative mass spectrometry. Proteomics 11, 535-553 (2011).

28. C. I. Carlström et al., (Per)chlorate-reducing bacteria can utilize aerobic and anaerobic pathways of aromatic degradation with (per)chlorate as an electron acceptor. mBio $\mathbf{6}$, e02287-14 (2015). 


\section{Acknowledgements}

242 Iowa) for providing comments on this manuscript, and Neil Fischer (UC Berkeley) for critically

243 reading the manuscript. We also thank Dr. Hwan Kim (Stony Brook University) for kindly

244 providing the O-antigen antibody and for fruitful discussions. We are also grateful for the

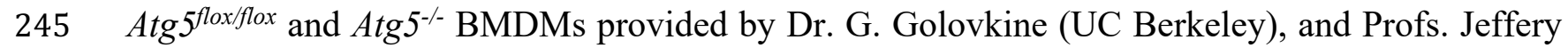

246 S. Cox and Michael Rape (both UC Berkeley) for fruitful discussions. Funding: P.E. was

247 supported by a postdoctoral fellowship from the Sweden-America Foundation. M.D.W. was

248 supported by NIH/NIAID grants R01 AI109044 and R21 AI138550. A mass spectrometer used in

249 this study was purchased with support from NIH grant 1S10 OD020062-01. Author

250 contributions: P.E. conceived the study with the assistance of M.D.W. P.E. performed laboratory

251 work and analysis, except for mass spectrometry, which was conducted together with A.T.I, and

252 animal experiments, which were conducted by T.P.B. P.E. drafted the initial manuscript, and A.T.I,

253 T.P.B, and M.W.D provided editorial feedback. Data and materials availability: All data used

254 in the analysis are provided in the main text or supplementary materials. Materials are available 255 upon request. 
bioRxiv preprint doi: https://doi.org/10.1101/2020.11.20.392290; this version posted December 10, 2020. The copyright holder for this preprint

(which was not certified by peer review) is the author/funder, who has granted bioRxiv a license to display the preprint in perpetuity. It is made available under aCC-BY-NC-ND 4.0 International license.

A

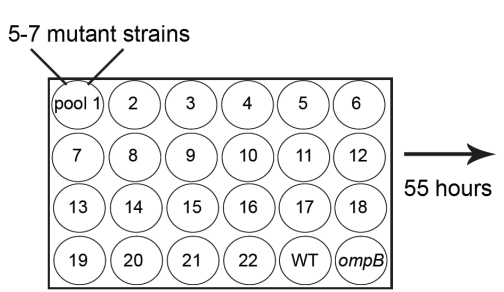

B

R. parkeri
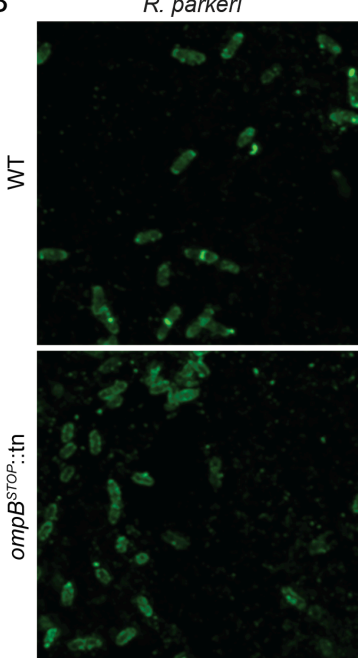

:

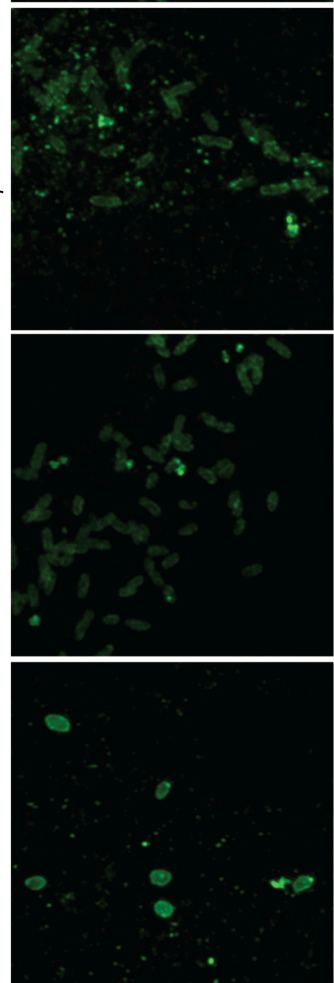

Polyubiquitin

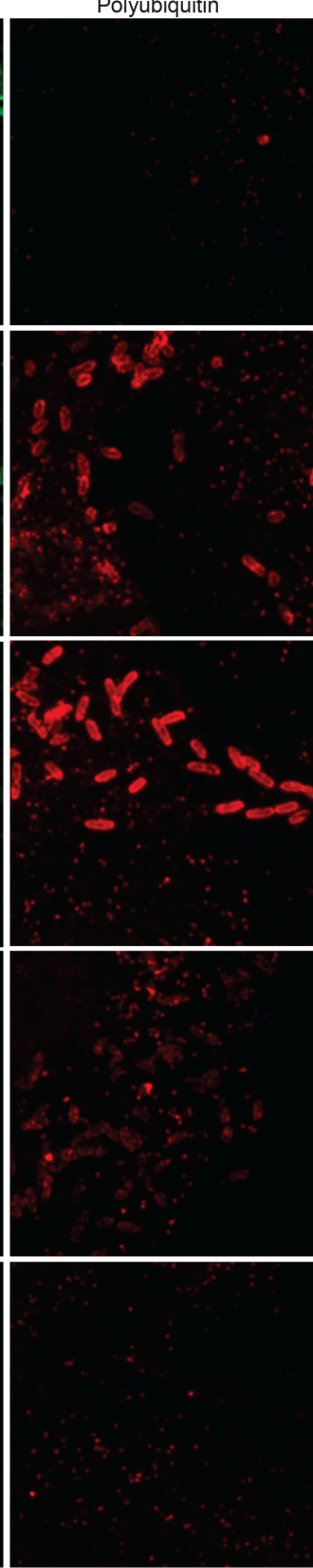

R. parkeri

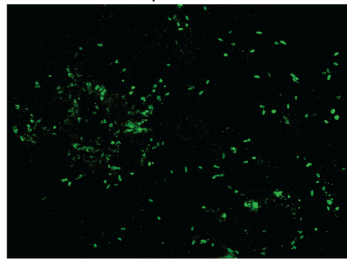

Merge
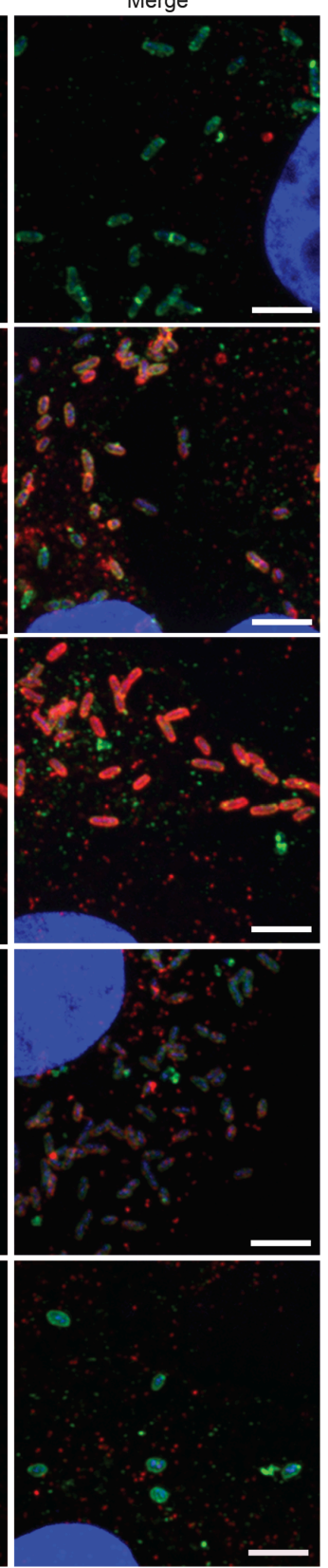

Polyubiquitin
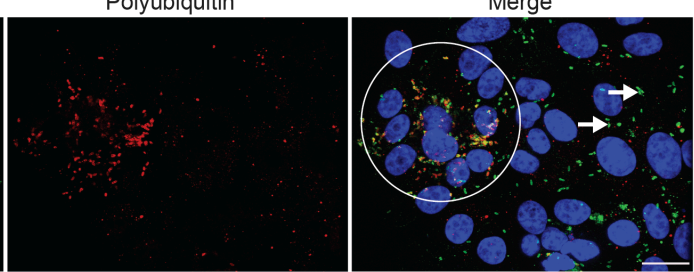

C

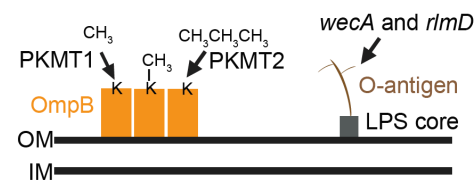

$\mathrm{D}$

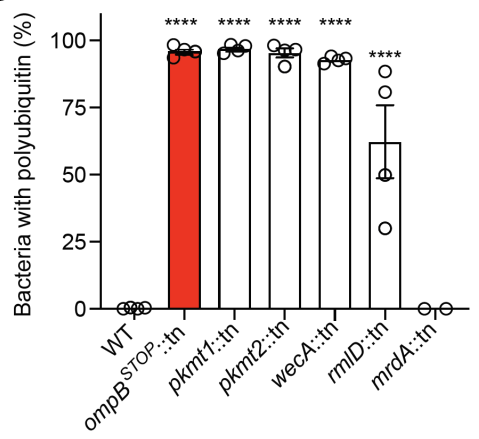

E

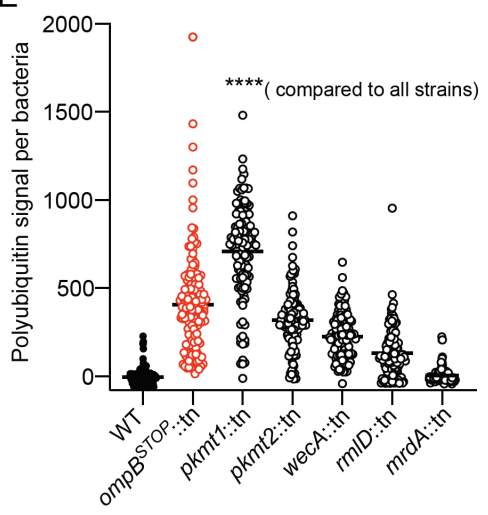

$F$

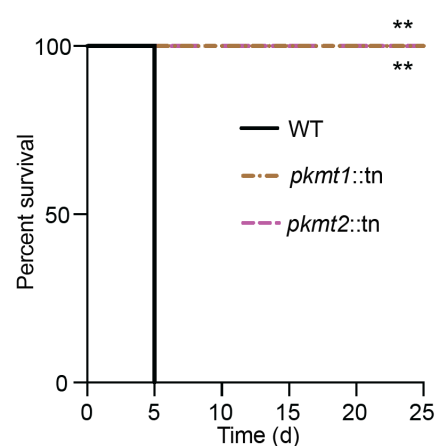


260 negative control. Circle, pUb-positive bacteria; arrows pUb-negative bacteria. Scale bar, $20 \mu \mathrm{m}$.

261 (B) Infected cells at 72 hours post-infection (h.p.i.) stained as in A. Scale bar, $5 \mu \mathrm{m}(n=4)$. (C)

262 Biological function of the genes identified. (D) Percentage of bacteria co-localized with pUb at 72

263 h.p.i. (Data are the mean \pm s.e.m.; $n=4$ for WT and mutant bacteria; $m r d A:: t n ; n=2$ ). Statistical

264 comparisons between WT and mutants were performed using a one-way ANOVA with Dunnett's

265 post-hoc test; $* * * * P<0.0001$. (E) pUb signal per bacteria. (Lines indicate the means; $n=3$ fields

266 of vision). Statistical comparisons were performed using a Kruskal-Wallis test with Dunn's post-

267 hoc test; $* * * * P<0.0001$. (F) Survival of Ifnar ${ }^{-/}$Ifngr $r^{-/}$mice intravenously infected with $5 \times 10^{6}$

268 WT or mutant bacteria ( $n=5$ mice, WT; $n=6$ mice, pkmt1::tn and pkmt2::tn). Statistical

269 comparison between WT and mutants was performed using a two-way ANOVA; ** $P<0.01$. 
A

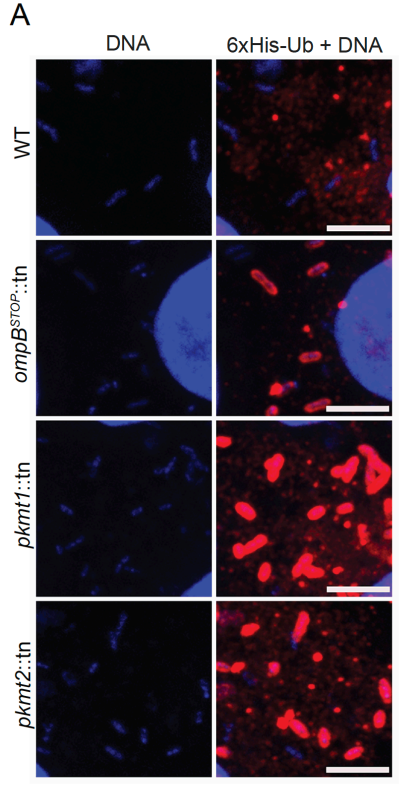

B $6 x$

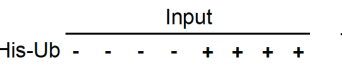

Pull down

WT + - - - + - - - + - - + - -

pknth- + - - + -

pkmt1::tn - - + - - - + - - - + - - - +

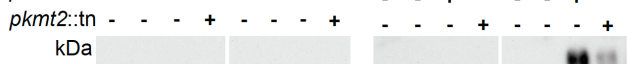

250 .

150

$\overrightarrow{100}$.

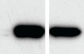

100 .

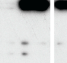

250

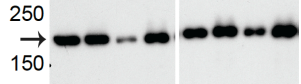

250
100
75

50

37

Fig. 2. Lysine methylation protects OMPs from ubiquitylation. (A) Micrographs of infected Vero cells expressing 6xHis-ubiquitin stained with anti-His antibody (red) and Hoechst (blue, bacterial and host DNA), at 28 h.p.i. Scale bar, $5 \mu \mathrm{m}$ (representative of $n=2$ ).

(B) Western blot of His-Ub input and pull-down samples from infected control and 6xHis-ubiquitin expressing cells, probed for OmpB, OmpA, and $\mathrm{pUb}$ (representative of $n=3$ ).

(C) pUb-enriched (TUBE-1, pan specific) samples from purified bacteria probed for OmpB, OmpA, and OmpW (OmpB and OmpA of endogenous molecular weight represent non-specific binding to TUBE-1 beads) (representative of $n=3$ ). Asterisks indicate OmpB and OmpA that exhibit increased molecular weight, indicating ubiquitylation. Arrows indicate OmpB and OmpA of endogenous molecular weight. 

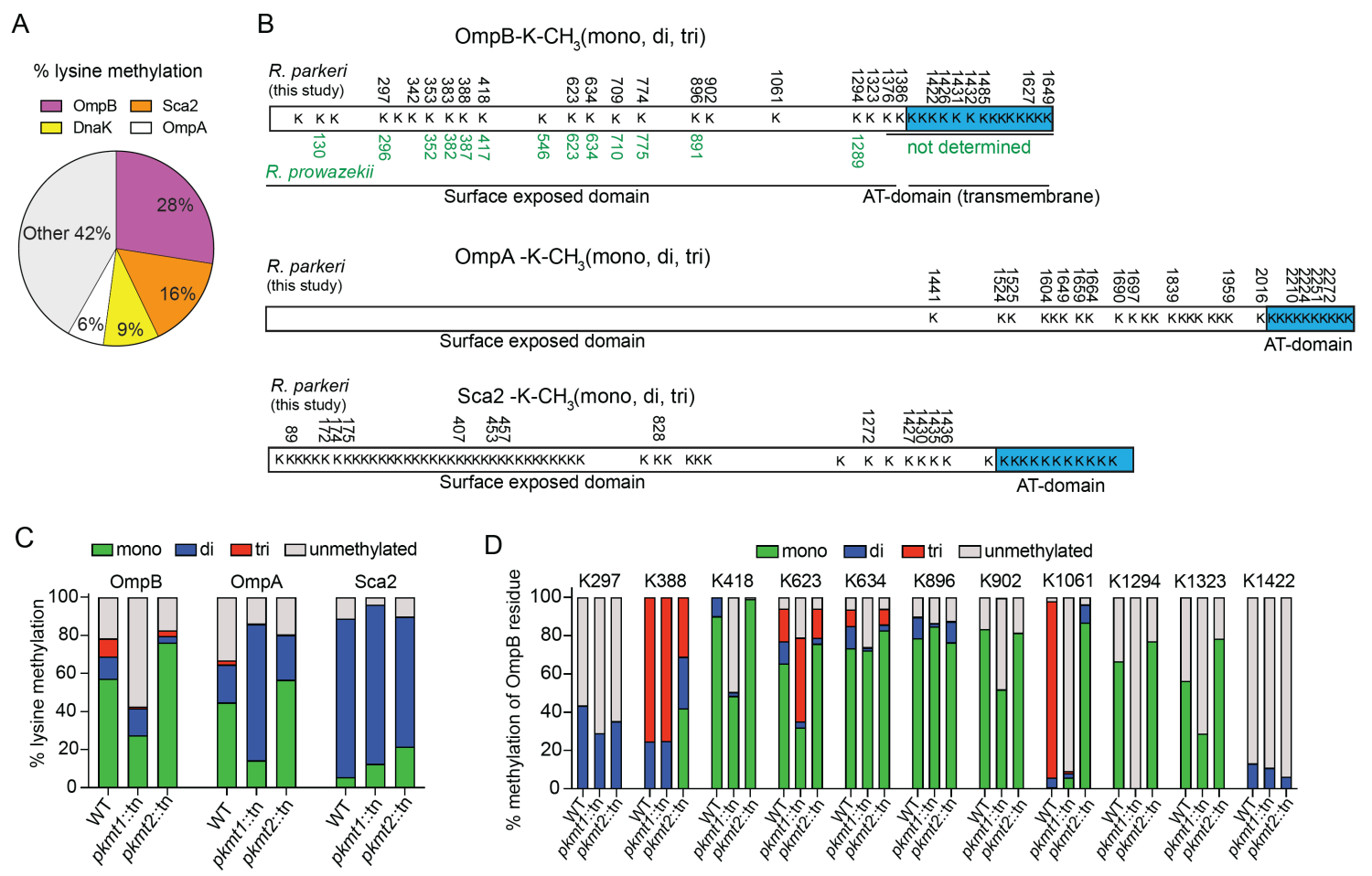

E
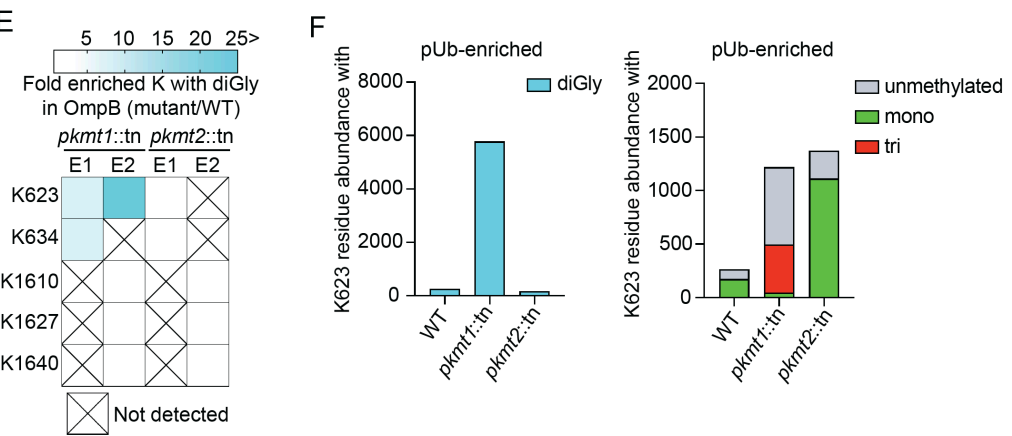

Fig. 3. Methylation camouflages lysines from ubiquitylation. (A) Percentage lysine methylation abundance of total among the abundantly detected proteins in WT $R$. parkeri determined by LCMS (data are combined from $n=2$ ). (B) Methylated lysines $(\mathrm{K})$ are indicated with residue number in each OMP, and lysines in R. prowazekii OmpB known to be methylated in green (17). K78, $\mathrm{K} 131, \mathrm{~K} 149, \mathrm{~K} 312$, and $\mathrm{K} 547$ in OmpB were unmethylated $(n=5$, using data-independent and data-dependent acquisition modes). (C) Percentage of total abundances of the lysines methylated in $\mathbf{B}$, that is unmethylated, mono-, di-, or tri-methylated (data are the mean of $n=2$ ). (D) Percentage of individual lysines in OmpB that are unmethylated, mono-, di- or tri-methylated. Only residues repeatedly detected in all strains were analyzed (data are the mean of $n=2$ ). (E) Heat map representation of OmpB residues from $p k m t 1:$ :th or $p k m t 2::$ th that have similar levels of K-diGly peptides (white boxes) compared to WT, or with a 5-fold, or more, increase of K-diGly peptides (cyan) after pUb-enrichments $(n=2)$. (F) Abundances of ubiquitylated (diGly), unmethylated, mono-, di-, or tri-methylated OmpB K623 (data are the mean of $n=2$ ). Each experiment $(n)$ was performed in technical triplicate. 
A

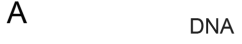

DNA

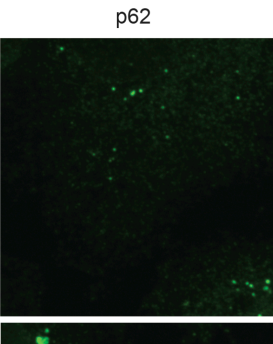

与

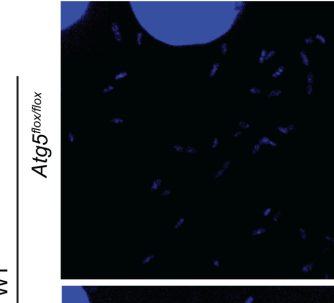

离

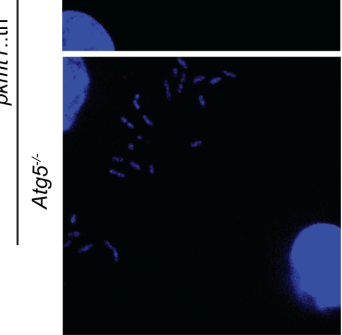

Polyubiquitin

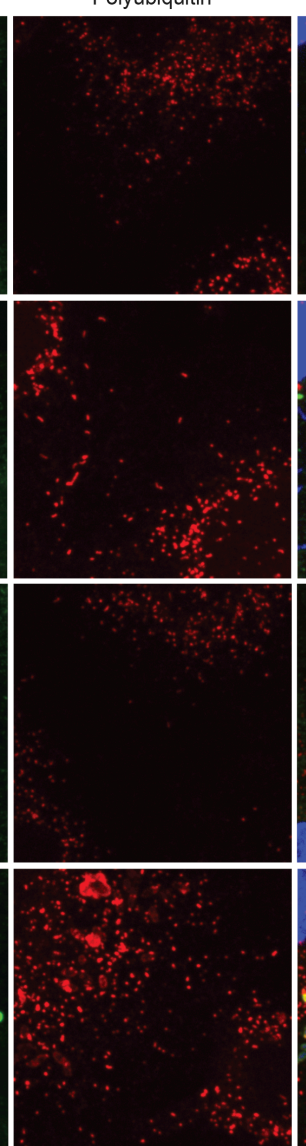

Merge
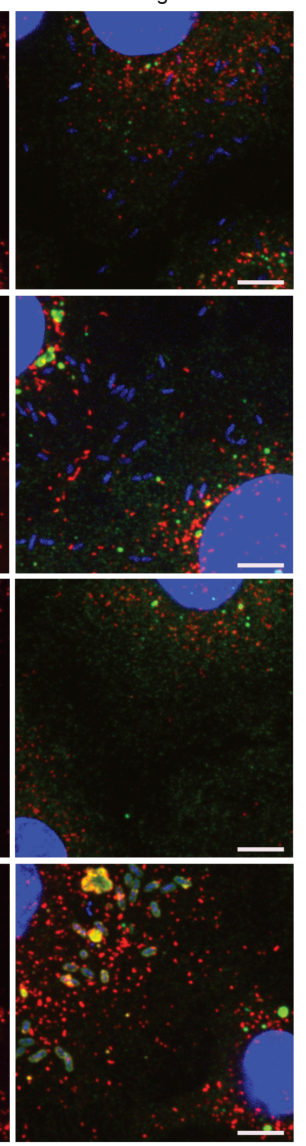

B

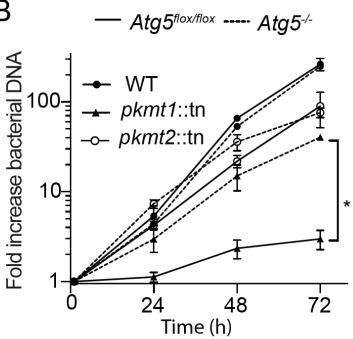

C
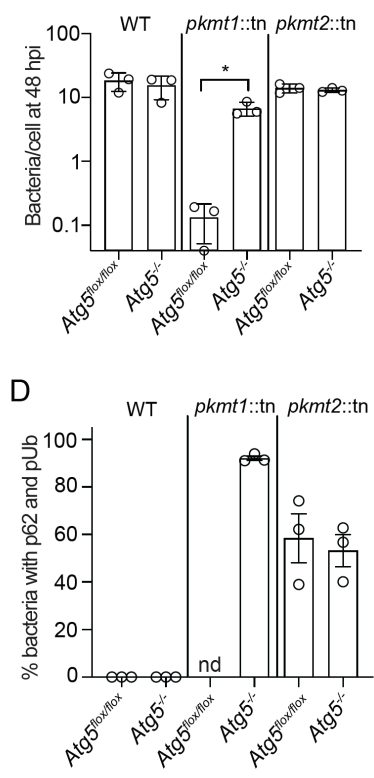

Fig. 4. Methylation prevents ATG5-dependent $R$. parkeri killing in macrophages. (A)

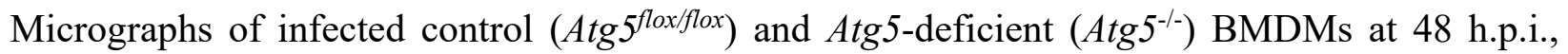
stained for DNA (blue), pUb (red), and p62 (green). Arrow indicate a bacterium positive for both $\mathrm{pUb}$ and p62. Scale bars, $5 \mu \mathrm{m}(n=3)$. (B) Bacterial growth curves in control and Atg5 $5^{-/} \mathrm{BMDMs}$, as measured by genomic equivalents using qPCR $(n=3)$. (C) Quantification of the mean number of bacteria per cell $(n=3)$.

(D) Quantification of the percentage of bacteria with p62 and pUb ( $n$

$302=3$ ). nd, not determined. Statistical comparisons in $\mathbf{B}$ and $\mathbf{C}$ were performed using a Brown303 Forsyth and Welch ANOVA with Dunnett's post-hoc test; ${ }^{*}, P<0.05$. Presented data are the mean $304 \pm$ s.e.m. 


\section{Supplementary Figures}

A OmpA
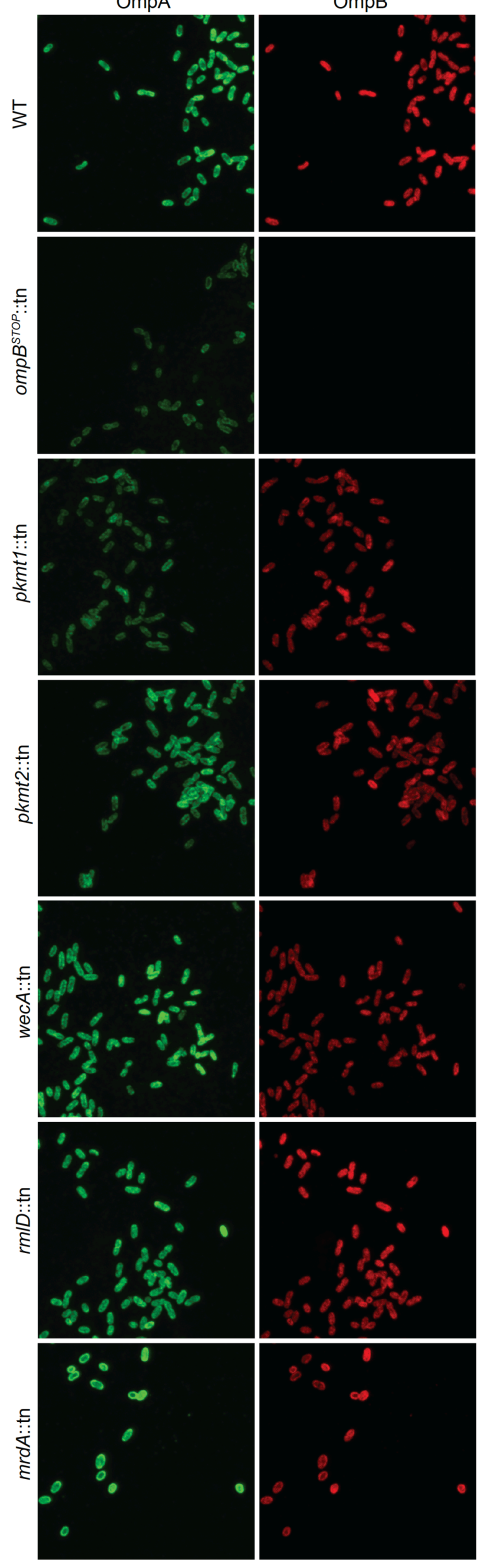

DNA
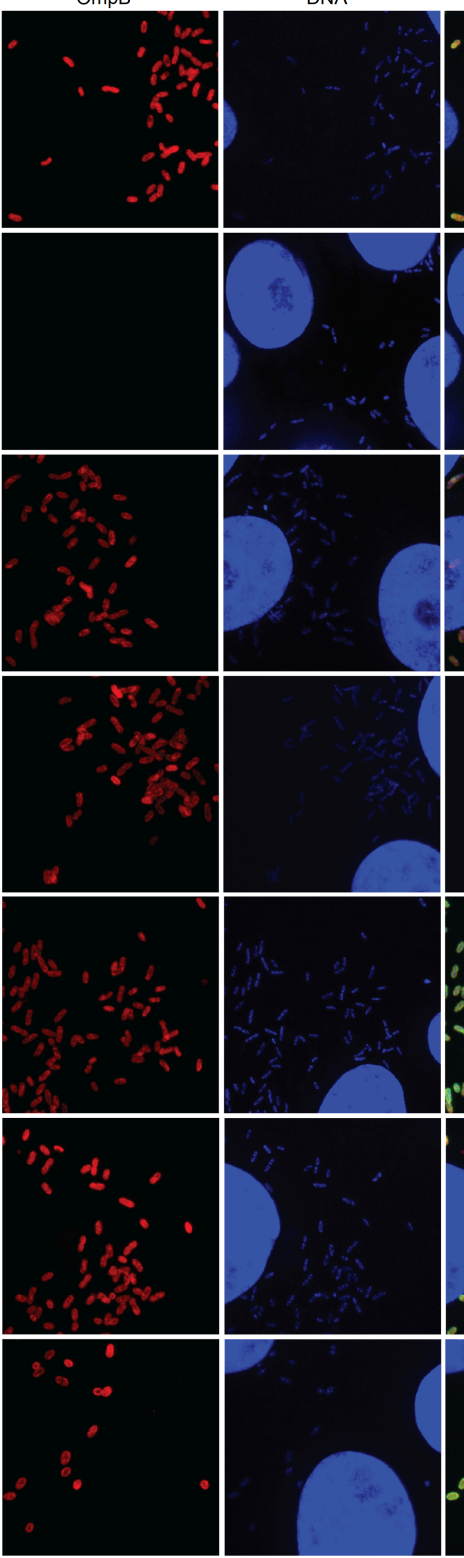

Merge
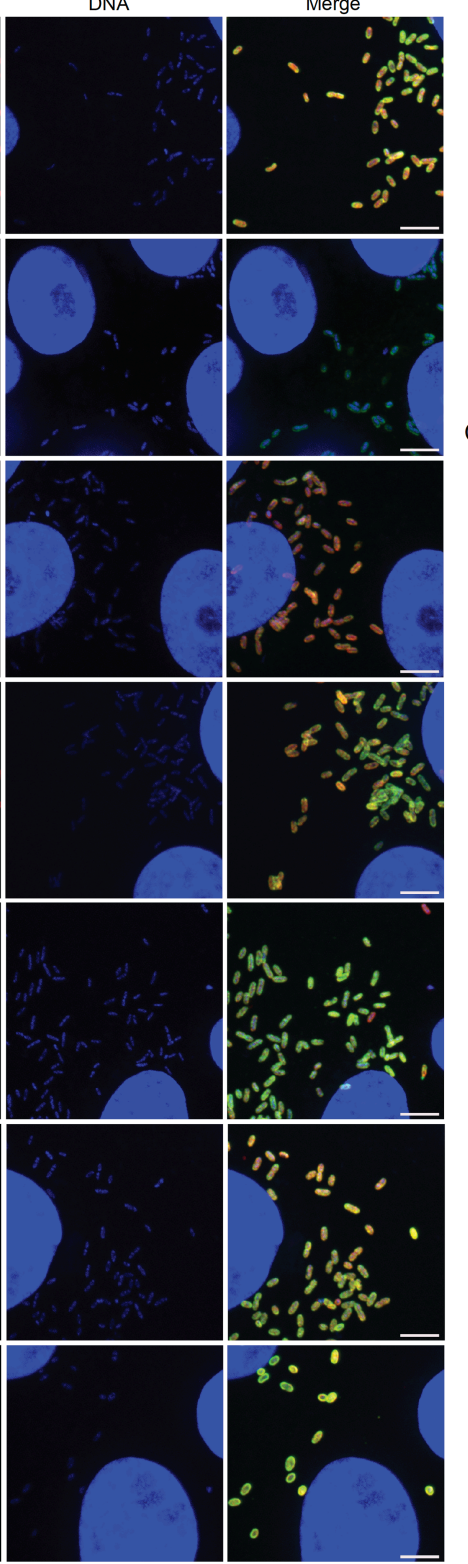

B
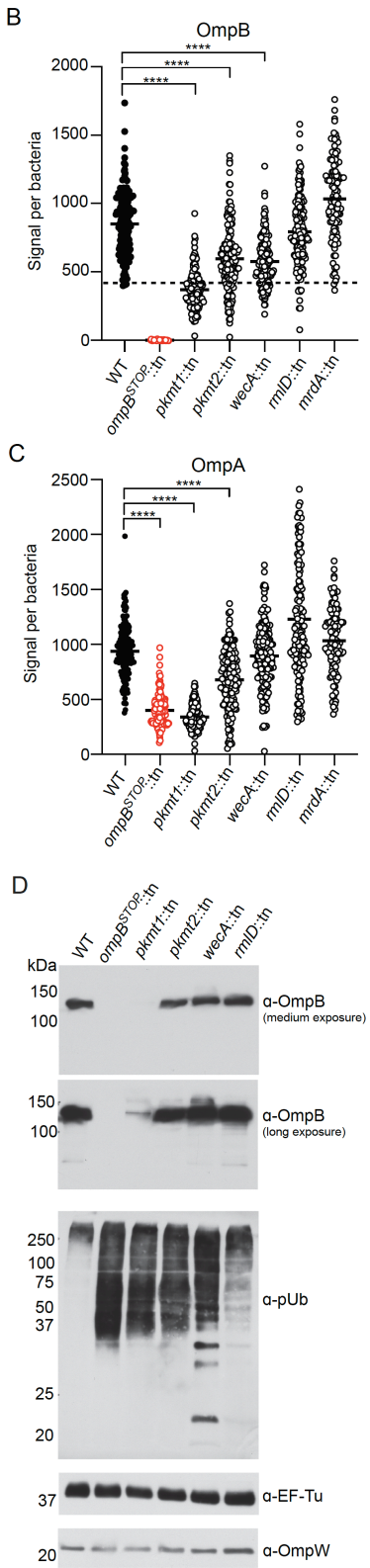

Fig. S1. Polyubiquitylated mutant $R$. parkeri strains are positive for OmpA and OmpB. (A) Micrographs of Vero cells infected with the indicated strains stained for OmpA (green, 13-3 antibody), OmpB (red, OmpB-antibody), and DNA (blue, Hoechst) at 72 h.p.i. (representative of $n=3$ ). Scale bar $5 \mu \mathrm{m}$. (B) Quantification of OmpB signal per bacteria. (Lines indicate the means; $n=3$ fields of vision; $\geq 50$ bacteria per field of vision were analyzed). Statistical comparisons were performed using a Kruskal-Wallis test with Dunn's post-hoc test; **** $P<0.0001$ between indicated strains. Dashed line indicates that the majority of mutant bacterial populations, except pkmt 1::tn bacteria, have OmpB levels comparable to WT bacteria. (C) Quantification of OmpA signal per bacteria as in B. (D) Western blot of $5 \times 10^{6}$ purified bacteria probed for OmpB, pUb, OmpW and EF-Tu (bacterial loading controls) (representative of $n=2$ ). 

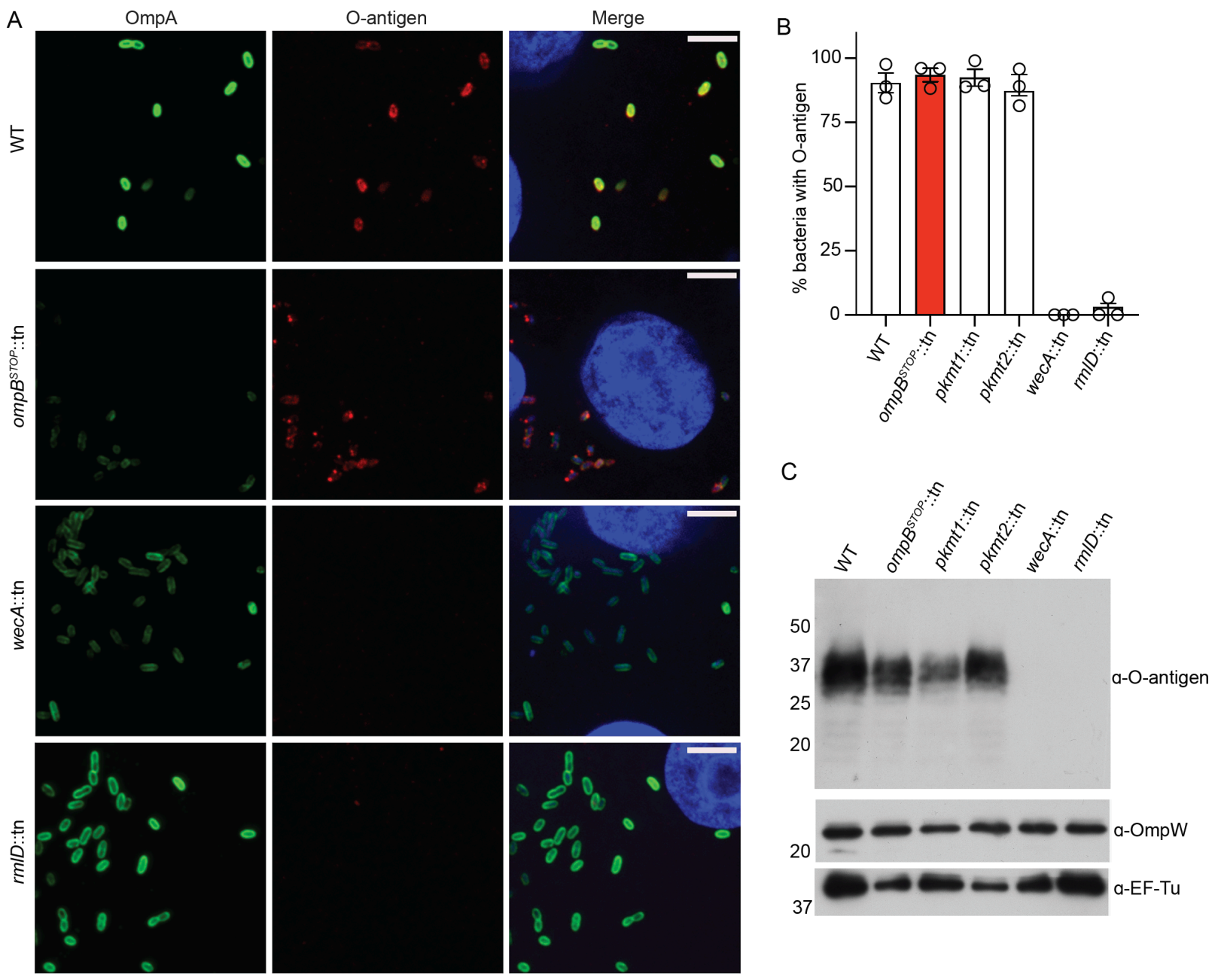

Fig. S2. The wec $A$ ::tn and $r m l D:$ :tn bacteria lack the O-antigen. (A) Micrographs of Vero cells infected with the indicated strains stained for OmpA (green, 13-3 antibody), the O-antigen (red,

$3225 \mu \mathrm{m}$. (B) Percentage of bacteria positive for the O-antigen at 72 h.p.i. $n=2 ; \geq 90$ bacteria were counted in each infection (data are the mean \pm s.e.m.; $n=3$ ). (C) Western blot of $5 \times 10^{6}$ purified

324 WT and mutant bacteria probed for the O-antigen. OmpW and EF-Tu were used as bacterial 325 loading controls (representative of $n=3$ ). 


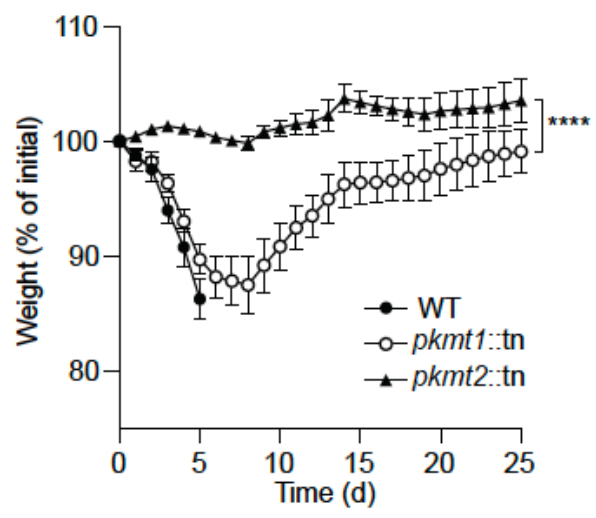

327 Fig. S3. PKMT1 plays a more significant role in causing disease in vivo compared to PKMT2.

328 (A) Weight changes of Ifnar ${ }^{-/}$Ifngr $r^{-/}$mice intravenous infected with $5 \times 10^{6} \mathrm{WT}$, pkmt $1:$ tn, or 329 pkmt2::tn bacteria (data are the mean \pm s.e.m. $n=5$, WT; $n=6, p k m t 1::$ tn and $p k m t 2::$ tn, combined 330 from two independent experiments). A two-way ANOVA from 0 to 25 days post-infection (d.p.i.) 331 was used to statistically compare the weight changes between the pkmt $1:$ tn and pkmt2::tn mutants. 

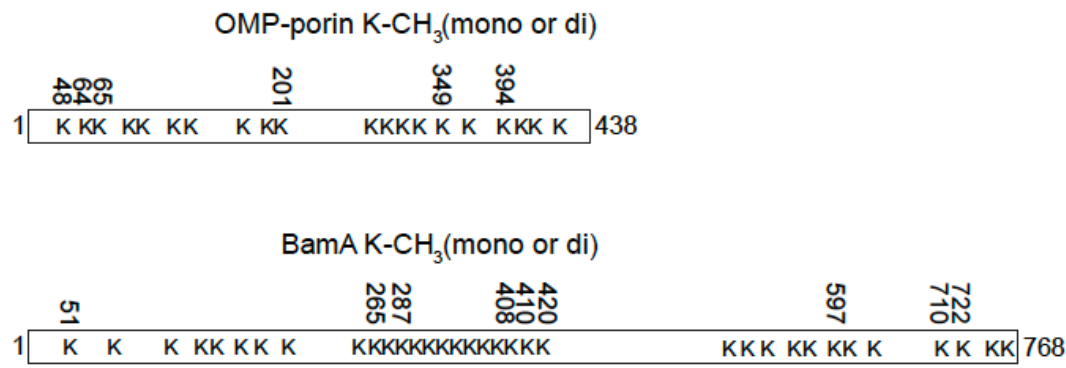

334 Fig. S4. Lysine-methylome reveals that $R$. parkeri OMP-porin, BamA, and the released factor residues without, determined by LC-MS as in Fig. 3B (data is combined from five independent experiments). See also Table S2. 


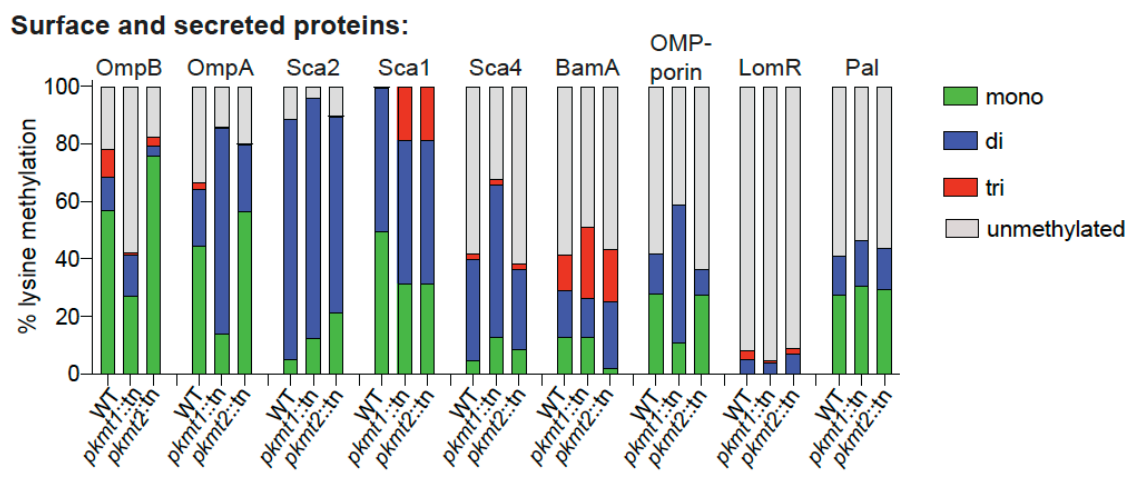

Chaperone and translational proteins:

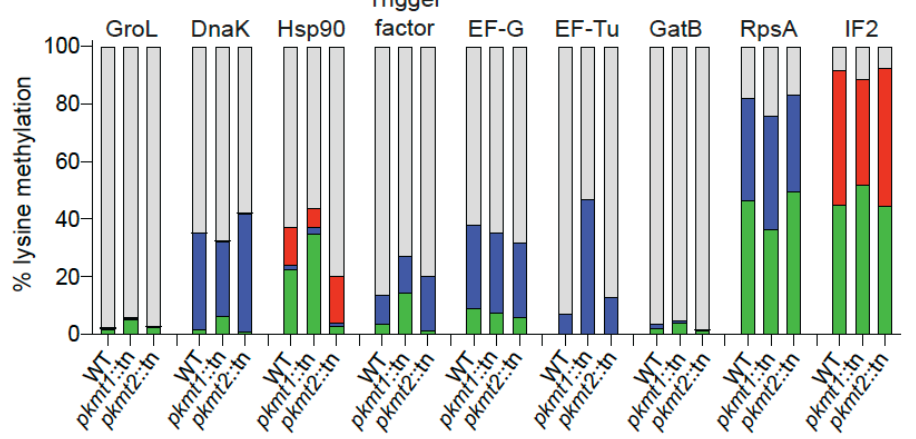

Metabolic proteins:

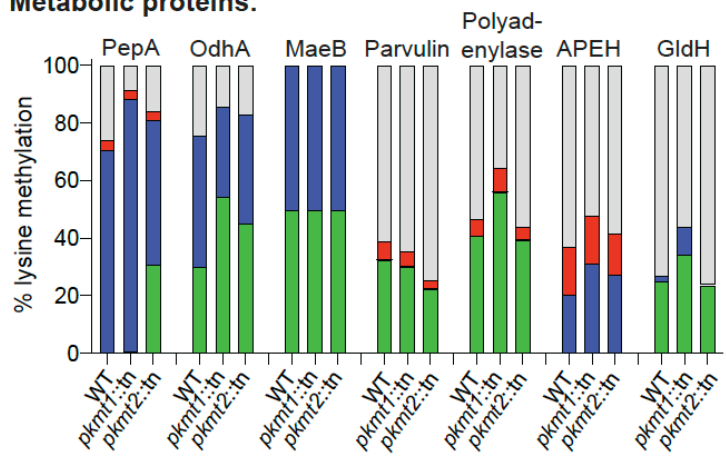

Hypothetical $(\mathrm{H})$ and other proteins:

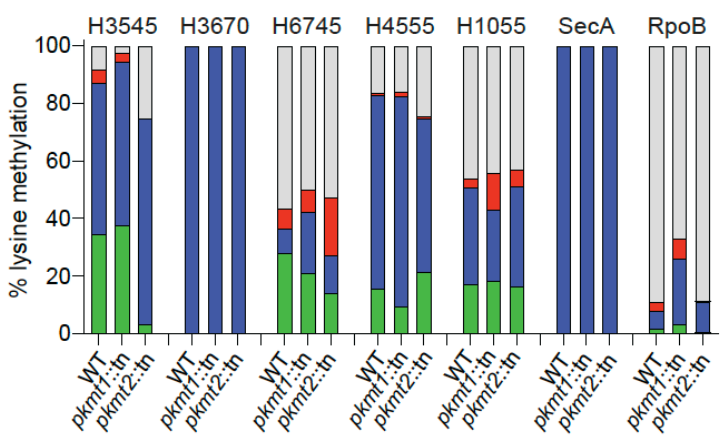

Fig. S5. PKMT1 primarily modifies bacterial OMPs. Percentage of total abundances of the lysines methylated in WT, that is unmethylated, mono-, di-, or tri-methylated in respective strain. Proteins with $\geq 3$ lysines methylated detected in independent experiments are shown (mean of $n=$ 2, performed in technical triplicates). OmpB, OmpA and Sca2 data are the same as in Fig. 3C. See also Table S2. 


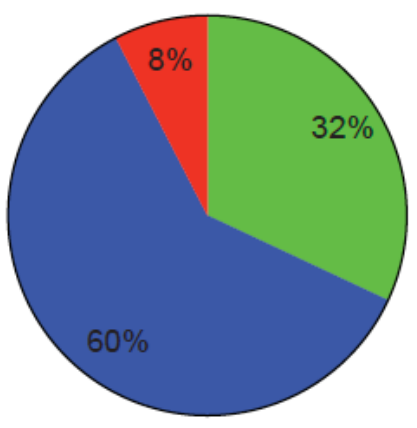
proteins. Percentage of total lysines abundances detected to as mono-, di-, or tri-methylated in 348 triplicates). 
A

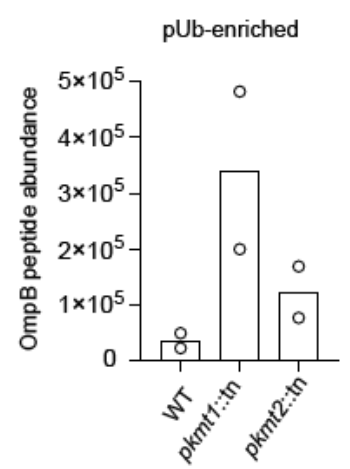

B methylation is reduced. (A) OmpB peptide abundance values after pUb-enrichments from respective strain, as determined by LC-MS (data are mean of $n=2$, performed in triplicates). (B) Abundance values of lysines in OmpB that are ubiquitylated (diGly), unmethylated, mono-, di-, or tri-methylated, in respective strain after pUb-enrichments as in Fig 2C. Only residues detected in independent experiments are shown (data are the mean of $n=2$, performed in technical triplicates). K623 data in $\mathbf{B}$ are the same as in Fig. 3F. 
DNA

p62

NDP52 Merge
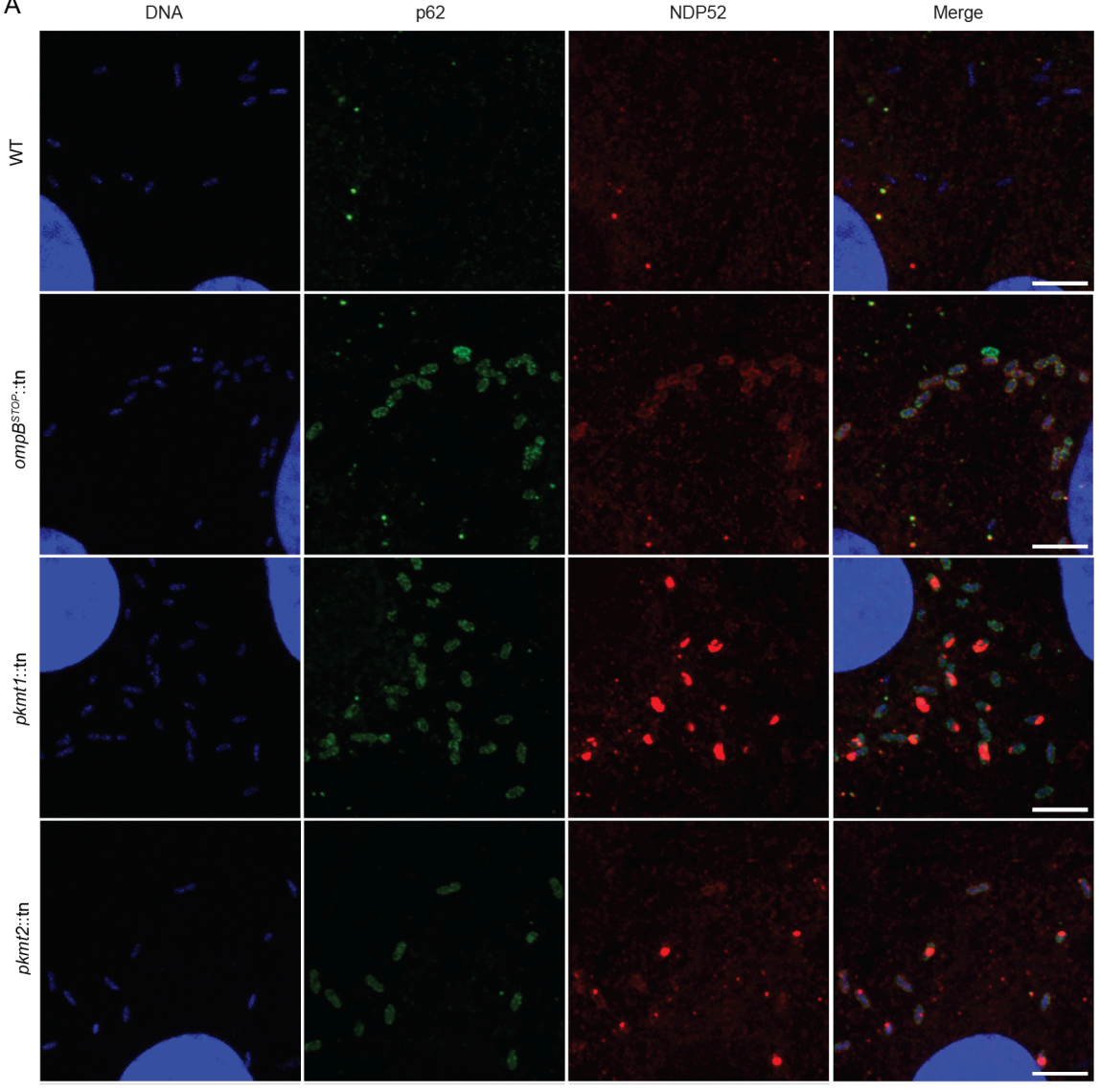

B

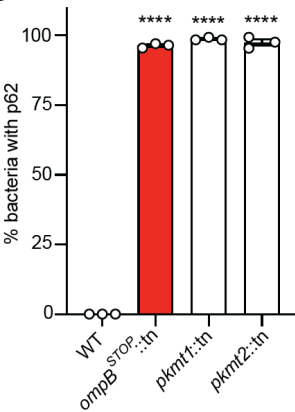

C

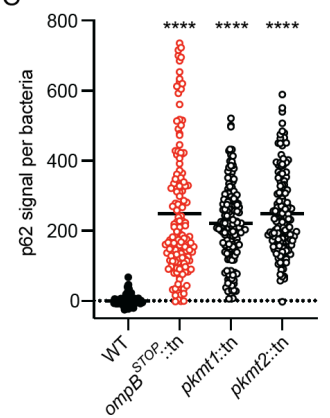

D

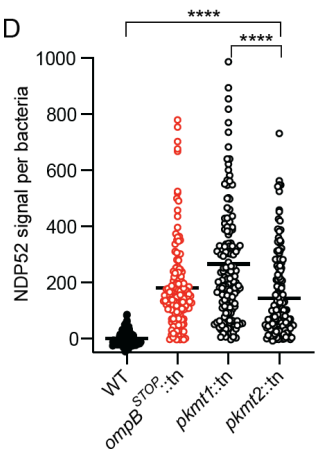

Fig. S8. OmpB and lysine methylation block recruitment of autophagy receptors to $R$. parkeri. (A) Micrographs of Vero cells infected with the indicated strains: stained for bacterial and host DNA (blue, Hoechst), p62 (green, p62-antibody), and NDP52 (red, NDP52-antibody) at 72 h.p.i ( $n=4$ for p62 staining; $n=2$ for NDP52 staining). (B) Percentage of bacteria that show rimlike surface localization of p62 at 72 h.p.i. (Data are the mean \pm s.e.m.; $n=3 ; \geq 142$ bacteria were counted for each infection). Statistical comparisons between WT and ompB ${ }^{S T O P}:: \mathrm{tn}$, pkmt1::tn, $p k m t 2::$ tn were performed using a one-way ANOVA with Tukey's post-hoc test; $* * * * P<0.0001$. (C) Quantification of p62 signal per bacteria from a representative experiment. Lines indicate the means. ( $n=3$ fields of vision; $\geq 50$ bacteria per field of vision were analyzed). Statistical comparisons were performed using a Kruskal-Wallis test with Dunn's post-hoc test; **** $P<$ 0.0001 between indicated strains. (D) Quantification of NDP52 signal per bacteria as in C. 
bioRxiv preprint doi: https://doi.org/10.1101/2020.11.20.392290; this version posted December 10, 2020. The copyright holder for this preprint (which was not certified by peer review) is the author/funder, who has granted bioRxiv a license to display the preprint in perpetuity. It is made available under aCC-BY-NC-ND 4.0 International license.

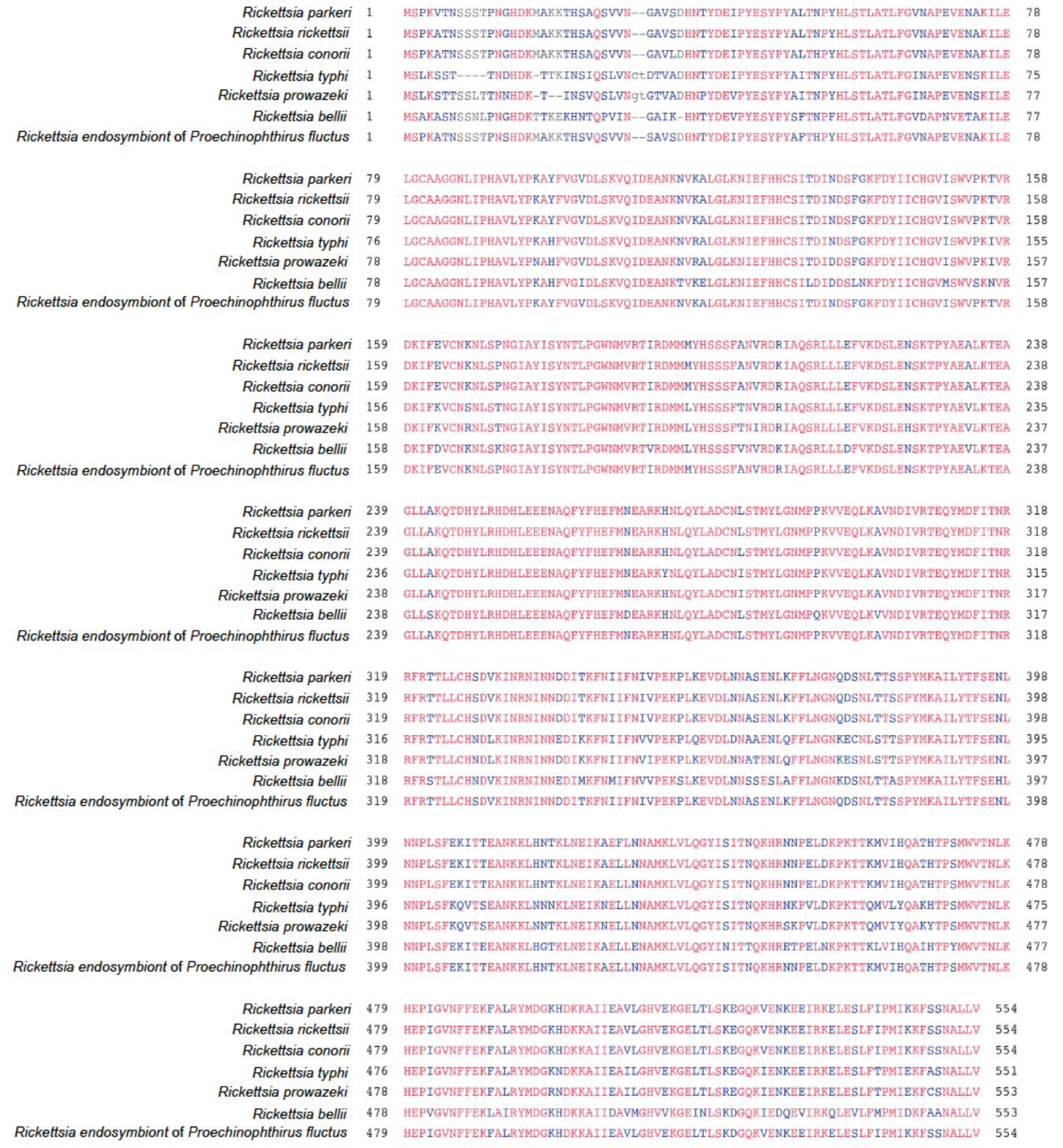

Rickettsia parkeri 1

Rickettsia typhi

Rickettsia parkeri 7

Rickettsia typhi 76

Rickettsia parkeri 159

Rickettsia typhi 15

Rickettsia endosymbiont of Proechinophthirus fluctus

Rickettsia parkeri 239

Rickettsia typhi 236

kettsia prowazeki 23

Rophthirus fluctus 239

Rickettsia conorii 3

Rickettsia bellii

Rickettsia rickettsii

Rickettsia conorii

Rickettsia bell

thirus fluctus

Rickettsia typhi

Rickettsia bellii

Fig. S9. The PKMT1 enzyme is highly conserved between diverse rickettsial species. Amino acid sequence alignment of PKMT1 from $R$. parkeri (WP 014411082.1), $R$. rickettsii (WP 012262600.1), R. conorii (WP 016926592.1), R. typhi (WP 011191207.1), R. prowazekii (WP 004596928.1), $R$. bellii (WP 045799810.1), and a Rickettsia endosymbiont (WP 062811822.1), using COBALT. Amino acids indicated in red are identical; blue, variation between species; grey, one or more of the analyzed proteins are lacking this residue(s). 
bioRxiv preprint doi: https://doi.org/10.1101/2020.11.20.392290; this version posted December 10,2020 . The copyright holder for this preprint (which was not certified by peer review) is the author/funder, who has granted bioRxiv a license to display the preprint in perpetuity. It is made available under aCC-BY-NC-ND 4.0 International license.

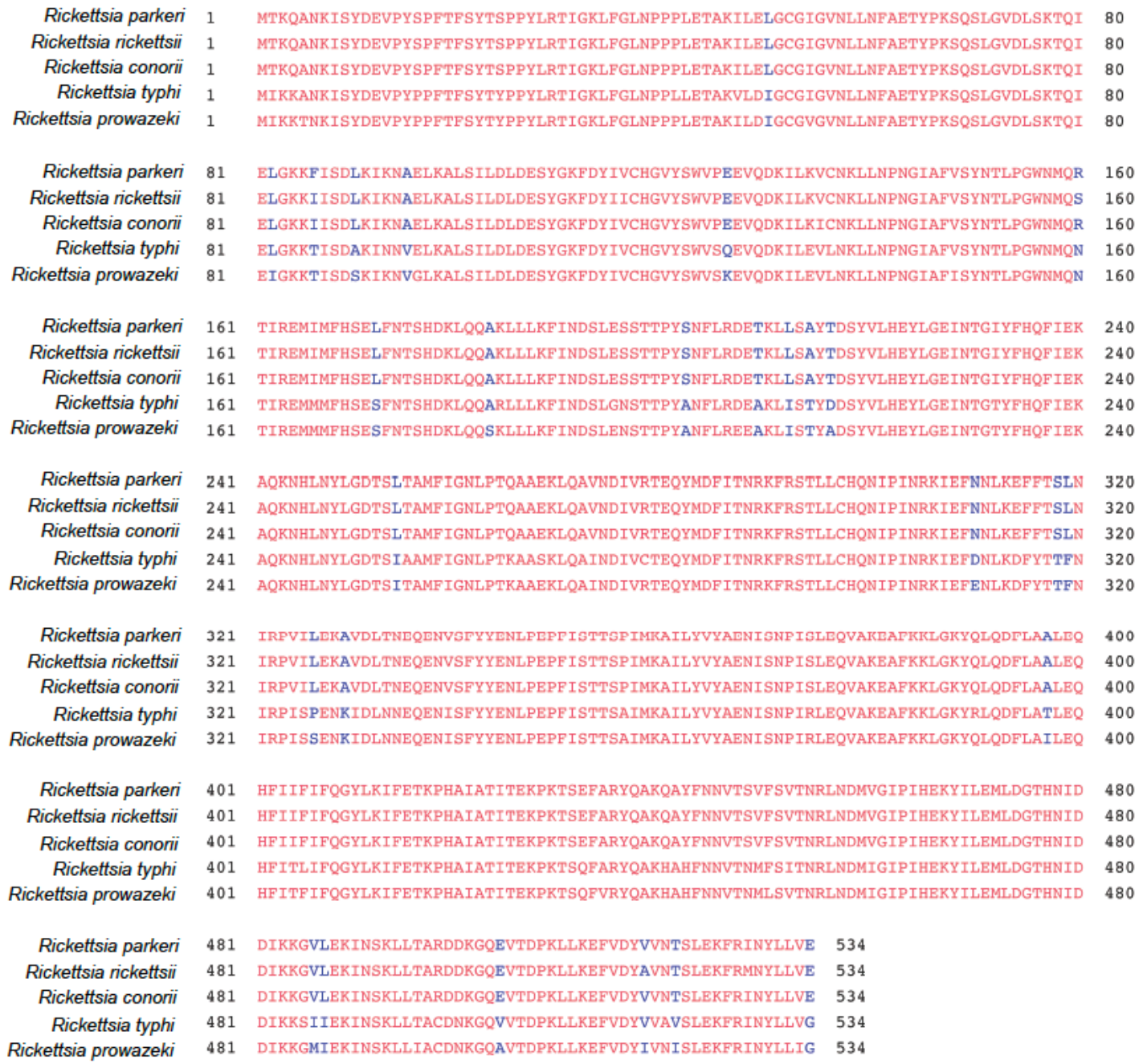
absent from $R$. bellii and the Rickettsia endosymbiont Proechinophthirus fluctus. Amino acid sequence alignment of PKMT2 from $R$. parkeri (WP 014410272.1), $R$. rickettsii (WP 012150259.1), R. conorii (WP 016925880.1), R. typhi (WP 011190574.1), and $R$. prowazekii (WP_004596662.1) using COBALT. PKMT1 of R. bellii and the Rickettsia endosymbiont Proechinophthirus fluctus showed 51\% identity to PKMT2 of $R$. parkeri using single alignment BLAST at NCBI; however, no PKMT2 variants could be found in these organisms, and therefore they were excluded from this analysis. Amino acids indicated in red are identical; blue, variation between species. 
bioRxiv preprint doi: https://doi.org/10.1101/2020.11.20.392290; this version posted December 10, 2020. The copyright holder for this preprint (which was not certified by peer review) is the author/funder, who has granted bioRxiv a license to display the preprint in perpetuity. It is made available under aCC-BY-NC-ND 4.0 International license.
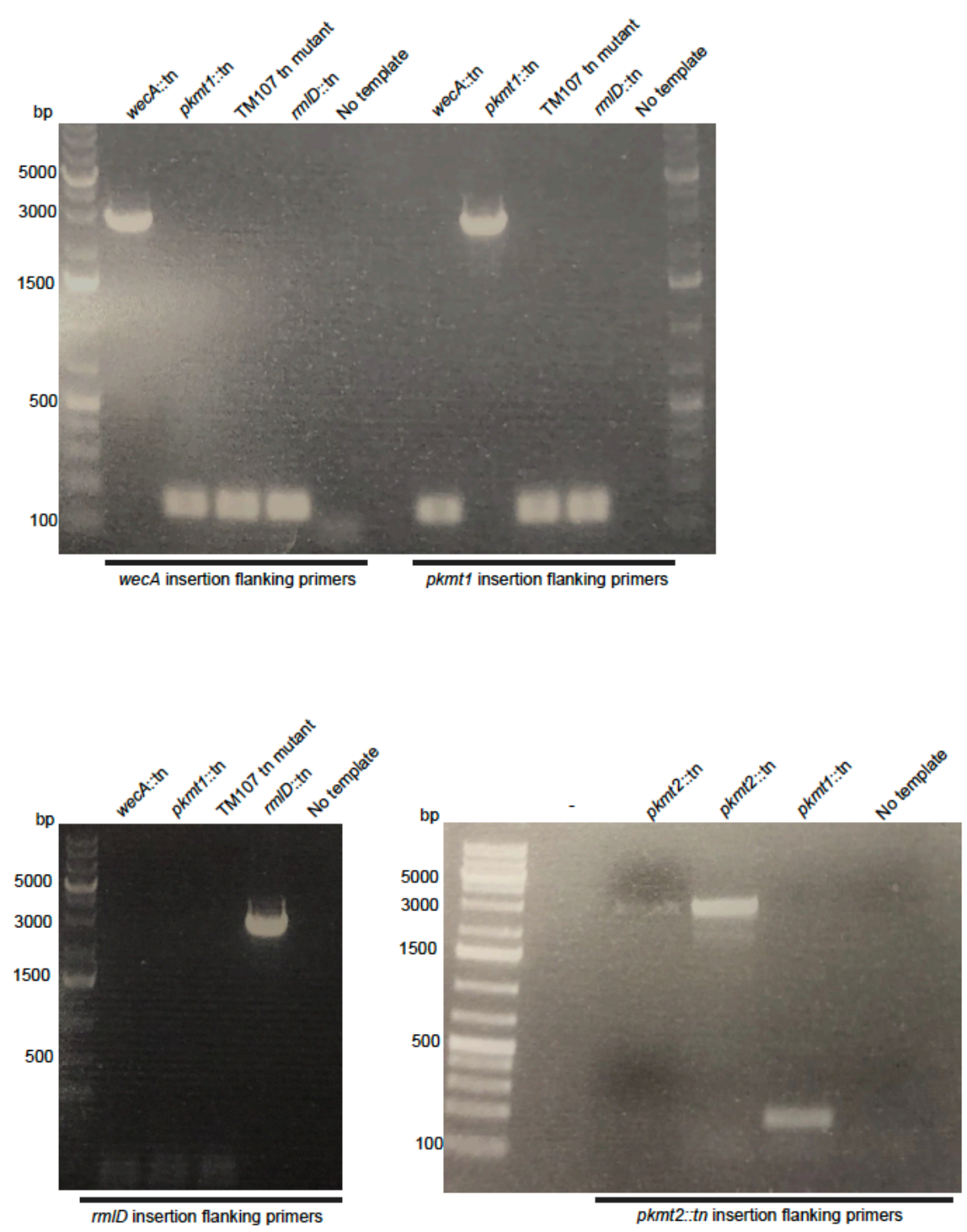

Fig. S11. Strain validation of clonality and insertion site using primers for flanking chromosomal regions $(n=1)$. 


\section{Materials and Methods}

\section{Cell lines and primary mouse macrophages}

Vero cells were purchased from the UC Berkeley Cell Culture Facility and the identity was repeatedly confirmed by mass-spectrometry analysis. Cells were grown at $37{ }^{\circ} \mathrm{C}$ and $5 \% \mathrm{CO}_{2}$ in DMEM (Gibco, cat. no. 11965) with high glucose $\left(4.5 \mathrm{~g} \mathrm{l}^{-1}\right)$ and 2\% heat-inactivated (30 min, 56 negative by DAPI staining and fluorescence microscopy screening at the UC Berkeley Cell Culture Facility.

BMDMs generated from the femurs of mutant Atg $5^{\text {flox } f l o x}$ and matched $A \operatorname{tg} 5^{-/}$C57BL/6 mice were a kind gift from the laboratory of Jeffery S. Cox (UC Berkeley), and they were prepared as previously described (11) although in the absence of antibiotics. Genotypes were confirmed by

400 PCR and Sanger sequencing at the UC Berkeley DNA Sequencing Facility, as previously 401 described (11).

\section{Rickettsia parkeri strain generation and validation}

WT R. parkeri strain Portsmouth (NCBI accession no. NC 017044.1; originally a gift from

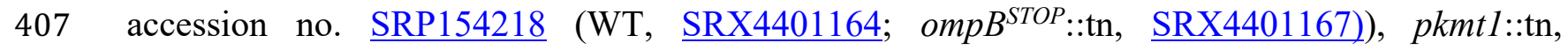

408 pkmt2::tn, wecA::tn and $r \operatorname{lm} D::$ tn mutants were propagated and purified every $\sim 6-10$ months as

409 described below. Side-by-side experimental comparisons were made between stocks prepared at 410 similar times. 
412 strains, screened for $\mathrm{pUb}$, were previously isolated in a screen for small plaque mutants $(13,22)$.

413 The $\operatorname{ompB}^{S T O P}:$ :tn was previously isolated in a suppressor screen and lacked expression of OmpB

414 (11). The $r m l D:: t n$, and 132 other transposon insertion mutant strains, screened for pUb, were

415 isolated in an independent screen in which mutants were isolated without regard for plaque size

416 (Table S1). The genomic locations of transposon insertion sites for all mutants were determined

417 by semi-random nested PCR. To verify the insertions and clonality, we used PCR reactions that

418 amplified the transposon insertion site using primers for flanking chromosomal regions:

419 5'GCTCACTAGATAGCACTCG'3 and 5'GCTCGATTTATCTCACTTTATG'3 for $r l m D:: t n$, 420 5'CGTTTAATAGTCCAGTTAATTTGT'3 and 5'CCGTCTATACCGTCCATAAAAT'3 for 421 wecA::tn, 5'GCATCGAAATAACCCTGAG'3 and 5'GCAAACTTCTCAAAGAAATTAACG'3

422 for pkmt1::tn, 5'GCTAAGAAATCTTCTAATTTGATATTTTAC'3 and

423 5'CGAAAATTTACCTGAGCCTT'3 for $p k m t 2:: \mathrm{tn}$, 5'CGACACATAATAGCACAAACTAC'3

424 and 5'GCGGAGGCGGTAGTAAAG'3 for $m r d A$ ::tn (Fig. S11).

426 Screening for pUb-positive strains

To prepare the mutant library for screening, passage 1 (P1) transposon insertion mutants

428 were amplified one time in Vero cells using 24-well cell culture plates. At 5-12 d.p.i, when 50-

$42970 \%$ of the infected cells appeared to be rounded up (as a sign of infection) by visual inspection

430 using a light microscope, cell culture media were completely removed, and cells were subsequently

431 lysed in $500 \mu \mathrm{L}$ cold sterile water for $2-3$ minutes (min). Next, $500 \mu \mathrm{L}$ of $2 x$ cold sterile brain-

432 heart-infusion (BHI) broth (BD Difco, 237500) was added to the lysed cells, resuspended, and P2

433 bacteria were transferred to cryogenic storage vials and frozen at $-80{ }^{\circ} \mathrm{C}$. 
To screen for pUb-positive strains, $10-40 \mu \mathrm{L}$ of each of five to seven P2 mutant bacterial strains were diluted in $1 \mathrm{~mL}$ of room temperature (RT) cell culture media supplemented with $2 \%$ FBS. Subsequently, the pooled bacterial suspension was centrifuged at $250 \mathrm{~g}$ for 4 min at RT onto confluent Vero cells grown on coverslips in 24-well plates. Cells were then incubated at $33{ }^{\circ} \mathrm{C}$ and fixed at 50-55 h.p.i. with pre-warmed 4\% paraformaldehyde (PFA) (Ted Pella Inc., 18505) for 10 min at RT. If cells were over-infected (i.e., individual infection foci had grown together) as determined by immunofluorescence microscopy, infections of that specific pool were repeated

441 using reduced volumes of P2 bacteria. Next, fixed cells were permeabilized with $0.2 \%$ Triton-X 442 for 5 min and then stained with the anti-Rickettsia I7205 antibody (1:500 dilution; gift from Ted Hackstadt) and anti-polyubiquitin FK1 antibody (Enzo Life Sciences, BML-PW8805-0500; 1:250

444 dilution), followed by Alexa 488 anti-rabbit antibody (Invitrogen, A11008; 1:500 dilution) or goat 445 anti-mouse Alexa-568 (Invitrogen, A11004). Whole coverslips were manually inspected on a 446 Nikon Ti Eclipse microscope with 60x (1.4 numerical aperture) Plan Apo objective. The initial 447 screen revealed that five out of 39 mutant pools contained pUb-positive areas.

449 Vero cells, as stated above. Infected cells were also fixed and stained as above except that a post450 fixation step using 100\% methanol for 5 min was included and an OmpB antibody (11) and 451 Hoechst (Sigma, B2261, 1:2500 dilution) was used instead of the anti-Rickettsia I7205 antibody. 452 Samples were inspected as above and strains were scored as follows: 1) pUb-negative (49 strains), 453 2) a few infection foci were pUb-positive (2 strains: Sp mutant 24, insertion at bp position 753916; 454 Sp mutant 94, insertion at bp position 774831), 3) bacteria in the center of foci were pUb-positive 455 but not on the edges ( 2 strains: Sp mutant 43, insertion at bp position 651602-651604; Sp mutant 45645 , insertion at bp position 751156$), 4)$ almost all bacteria in all foci were pUb-positive (4 strains: 
457 pkmt1::tn, insertion at bp position 1161553 (gene MC1_RS06185); pkmt2::tn, insertion at bp 458 position $34100\left(M C 1 \_R S 00180\right)$; wecA::tn, insertion at bp position 1223170 (MC1_RS06510); and 459 rmlD::tn, insertion at bp position 455753 (MC1_RS02345).

\section{Rickettsia purification}

R. parkeri strains were propagated as described previously (11). "Purified bacteria" were

463 from five T175 flasks of Vero cells that after 5-8 days of infection (normally 75\% infected as

464 observed by light microscopy) were harvested in the media using a cell scraper. Bacteria were tehn 465 centrifuged $12000 \mathrm{~g}$ for $15 \mathrm{~min}$ at $4{ }^{\circ} \mathrm{C}$ in pre-chilled tubes. Pellets were resuspended in cold $\mathrm{K}-36$

466 buffer $\left(0.05 \mathrm{M} \mathrm{KH}_{2} \mathrm{PO}_{4}, 0.05 \mathrm{M} \mathrm{K}_{2} \mathrm{HPO}_{4}, \mathrm{pH}\right.$ 7, $100 \mathrm{mM} \mathrm{KCl}$ and $\left.15 \mathrm{mM} \mathrm{NaCl}\right)$ and a pre-chilled

467 dounce-homogenizer (tight fit) were used for 60 strokes to release bacteria from host cells. The

468 homogenate was then centrifuged at $200 \mathrm{~g}$ for $5 \mathrm{~min}$ at $4{ }^{\circ} \mathrm{C}$ to remove cellular debris. The 469 supernatant was overlaid onto cold 30\% v/v MD-76R (Mallinckrodt Inc., 1317-07) diluted in K-

47036 , and centrifuged at $58300 \mathrm{~g}$ for $20 \mathrm{~min}$ at $4{ }^{\circ} \mathrm{C}$ in an SW-28 swinging-bucket rotor. The bacterial 471 pellet was resuspended in cold $1 \times$ BHI broth ( $0.5 \mathrm{~mL} \mathrm{BHI}$ per infected T175 flask), and after letting 472 DNA precipitates sediment to the bottom of the tubes, bacterial suspensions were collected, 473 aliquoted and frozen at $-80^{\circ} \mathrm{C}$.

474 "Gradient-purified bacteria" were from ten T175 flasks of Vero cells, purified as above 475 with the addition of a 40/44/54\% v/v MD-76R (diluted in K-36 buffer) gradient step centrifuged 476 at $58300 \mathrm{~g}$ for $25 \mathrm{~min}$ at $4{ }^{\circ} \mathrm{C}$ using the SW-28 swinging bucket rotor. The bacteria were then 477 collected from the $44-54 \%$ interface, diluted in K-36 buffer, and pelleted by centrifugation at $47812000 \mathrm{~g}$ for $15 \mathrm{~min}$ at $4^{\circ} \mathrm{C}$. The pellet was resuspended in cold $1 \mathrm{x}$ BHI broth and subsequently 479 aliquoted and frozen at $-80^{\circ} \mathrm{C}$. 


\section{OmpW and EF-Tu antibody production}

The sequence encoding amino acids $22-224$ of outer membrane protein $\mathrm{W}$ (OmpW; WP_014411122.1) (a protein that lacks the signal peptide), or full-length Elongation factor Tu (EF-Tu; WP_004997779.1), were amplified by PCR from $R$. parkeri genomic DNA, and subsequently cloned into plasmid pETM1, which encodes N-terminal 6xHis and maltose-binding proteins (MBP) tags. From the resulting plasmids, fusion proteins were expressed in E. coli strain

BL21 codon plus RIL-Cam ${ }^{\mathrm{r}}$ (DE3) (QB3 Macrolab, UC Berkeley) by induction with $1 \mathrm{mM}$ isopropyl- $\beta$-D-thio-galactoside (IPTG) for 2-2.5 hours at $37^{\circ} \mathrm{C}$. Bacterial pellets were resuspended in lysis buffer $\left(50 \mathrm{mM} \mathrm{NaH}_{2} \mathrm{PO}_{4}, \mathrm{pH} 8.0,300 \mathrm{mM} \mathrm{NaCl}, 1 \mathrm{mM}\right.$ EDTA, and $1 \mathrm{mM}$ dithiothreitol (DTT)) and stored at $-80^{\circ} \mathrm{C}$. For protein purification, bacteria were thawed, lysozyme was added to $1 \mathrm{mg} / \mathrm{mL}$ (Sigma, L4919), and lysis was carried out by sonication. Lysates containing 6xHis-

492 MBP-OmpW and 6xHis-MBP-EF-Tu were incubated on amylose resin (New England Biolabs, 493 E8021L) (Qiagen, 1018244) and bound proteins were eluted in lysis buffer lacking EDTA and 494 DTT but containing $10 \mathrm{mM}$ maltose. Fractions were analyzed by SDS-PAGE and those with the 495 highest concentrations of fusion proteins were pooled to generate rabbit antibodies against OmpW 496 and EF-Tu. $1.2 \mathrm{mg}$ of purified 6xHis-MBP-OmpW and 6xHis-MBP-EF-Tu proteins were sent to

497 Pocono Rabbit Farm and Laboratory (Canadensis, PA), and immunization was carried out 498 according to their 91-d protocol.

\section{Western blotting}

To determine the levels of bacterial and host proteins in purified bacterial samples, $30 \%$ -

502 purified bacterial samples were boiled in 1x SDS loading buffer (150 mM Tris pH 6.8, 6\% SDS, 
$503 \quad 0.3 \%$ bromophenol blue, 30\% glycerol, $15 \% \beta$-mercaptoethanol) for $10 \mathrm{~min}$, then $5 \times 10^{6}$ PFUs

504 were resolved on an 8-12\% SDS-PAGE gel and transferred to an Immobilon-FL polyvinylidene

505 difluoride membrane (Millipore, IPEL00010). Membranes were probed for $30 \mathrm{~min}$ at room

506 temperature or $4^{\circ} \mathrm{C}$ overnight with antibodies as follows: affinity-purified rabbit anti-OmpB

507 antibody (11) diluted 1:200-30000 in TBS-T (20 mM Tris, $150 \mathrm{mM} \mathrm{NaCl,} \mathrm{pH} \mathrm{8.0,} \mathrm{0.05 \%} \mathrm{Tween}$

50820 (Sigma, P9416)) plus 5\% dry milk (Apex, 20-241); mouse monoclonal anti-OmpA 13-3

509 antibody diluted 1:10000-50000 in TBS-T plus 5\% dry milk; rabbit anti-OmpW serum diluted

510 1:8000 in TBS-T plus 5\% dry milk; mouse monoclonal FK1 anti-polyubiquitin antibody diluted

511 1:2500 in TBS-T plus 2\% BSA; rabbit anti-EF-Tu serum diluted 1:15000 in TBS-T plus 5\% dry

512 milk; or rabbit anti-O-antigen serum 1:5000 in TBS-T plus 5\% dry milk. Secondary antibodies

513 were: mouse anti-rabbit horseradish peroxidase (HRP) (Santa Cruz Biotechnology, sc-2357), or

514 goat anti-mouse HRP (Santa Cruz Biotechnology, sc-2005), all diluted 1:1000-2500 in TBS-T plus

$5155 \%$ dry milk. Secondary antibodies were detected with ECL Western Blotting Detection Reagents

516 (GE, Healthcare, RPN2106) for $1 \mathrm{~min}$ at room temperature, and developed using Biomax Light

517 Film (Carestream, 178-8207).

\section{Immunofluorescence microscopy}

$520 \quad$ R. parkeri infections were carried out in 24-well plates with sterile circle 12-mm coverslips

521 (Thermo Fisher Scientific, 12-545-80). To initiate infection, 30\%-purified bacteria were diluted in

522 cell culture media at room temperature to a MOI of 0.01 for Vero cells, and a MOI of 0.1 for

523 BMDMs. Bacteria were centrifuged onto cells at $300 \mathrm{~g}$ for $5 \mathrm{~min}$ at room temperature and

524 subsequently incubated at $33^{\circ} \mathrm{C}$. Next, infected cells were fixed for $10 \mathrm{~min}$ at room temperature

525 in pre-warmed $\left(37^{\circ} \mathrm{C}\right) 4 \%$ PFA diluted in PBS, pH 7.4, then washed 3 times with PBS. Primary 
526 antibodies were the following: for staining with the guinea pig polyclonal anti-p62 antibody

527 (Fitzgerald, 20R-PP001; 1:500 dilution), mouse polyclonal anti-NDP52 antibody (Novus

528 Biologicals, H00010241-B01P; 1:100 dilution), a rabbit anti-Rickettsia I7205 antibody (1:500

529 dilution; gift from Ted Hackstadt), or anti-polyubiquitin FK1 antibody (1:250 dilution), cells were

530 permeabilized with $0.5 \%$ Triton-X100 for 5 min prior to staining. For staining with mouse

531 monoclonal anti-OmpA 13-3 antibody (1:5000 dilution), anti-OmpB antibody (11) (1:1000

532 dilution), or rabbit anti-O-antigen serum (15) (1:500 dilution), infected cells were post-fixed in

533 methanol for 5 min at RT (no Triton-X). Cells were then washed 3 times with PBS and incubated

534 with the primary antibodies for $30 \mathrm{~min}$ at RT. To detect the primary antibodies, secondary goat

535 anti-rabbit Alexa-568 (Invitrogen, A11036), goat anti-mouse Alexa-568 (Invitrogen, A11004), or

536 goat anti-guinea pig Alexa-568 (Invitrogen, A11075), Alexa 488 anti-rabbit antibody (Invitrogen,

537 A11008; 1:500 dilution), Alexa 488 anti-mouse antibody (Invitrogen, A11001) antibodies were

538 incubated at room temperature for $30 \mathrm{~min}$ (all 1:500 in PBS with 2\% BSA). Images were captured

539 as $15-25$ z-stacks (0.1- $\mu \mathrm{m}$ step size) on a Nikon Ti Eclipse microscope with a Yokogawa CSU-XI

540 spinning disc confocal with 100x (1.4 NA) Plan Apo objectives, and a Clara Interline CCD Camera

541 (Andor Technology) using MetaMorph software (Molecular Devices). Images were processed

542 using ImageJ using z-stack average maximum intensity projections and assembled in Adobe

543 Photoshop. For quantification of the percentage of bacteria with pUb and p62, only bacteria that

544 co-localized with rim-like patterns of the respective marker were scored as positive for staining.

545 To quantify pUb, p62, NDP52, OmpB, and OmpA signal per bacteria, z-stacks were projected as

546 stated above, and the edges of individual bacteria were marked by the freehand region of interest

547 (ROI) function in ImageJ. Subsequently, the average pixel intensity within that ROI was measured.

$548 \mathrm{pUb} / \mathrm{p} 62 / \mathrm{NDP} 52$ signal intensities were calculated by subtracting the average pUb/p62/NDP52 
549 signal of WT bacteria from the $\mathrm{pUb} / \mathrm{p} 62 / \mathrm{NDP} 52$-value of each bacterium. OmpB signal intensity

550 was calculated by subtracting the average OmpB-signal of $\operatorname{omp} B^{S T O P}$ ::tn bacteria from the OmpB-

551 value of each bacterium. OmpA signal intensity was calculated by subtracting the average

552 background-signal (areas with no bacteria) from the OmpA-value of each bacterium.

554 Sample preparation for mass spectrometry to determine the lysine methylome

556 centrifuged at $11,000 \mathrm{~g}$ for $3 \mathrm{~min}$. Each pellet was resuspended in $50 \mu \mathrm{L}$ Tris $(10 \mathrm{mM})$-EDTA (10

$557 \mathrm{mM}$ ), $\mathrm{pH} \mathrm{7.6}$, and incubated for $45 \mathrm{~min}$ in a $45^{\circ} \mathrm{C}$ water bath. Bacterial surface fractions were

558 recovered from the supernatant after centrifugation at 11,000g for $3 \mathrm{~min}$. Pellet was resuspended

559 as above and incubated for additional $45 \mathrm{~min}$ at $45^{\circ} \mathrm{C}$ before resuspension in $50 \mu \mathrm{L}$ Tris $(10 \mathrm{mM})$ -

560 EDTA $(10 \mathrm{mM})$. Both pellet and surface fractions were boiled at $95^{\circ} \mathrm{C}$ for $10 \mathrm{~min}$. Samples were

561 cooled to RT prior to addition of $20 \mu \mathrm{L} 50 \mathrm{mM} \mathrm{NH}_{4} \mathrm{HCO}_{3}, \mathrm{pH} 7.5$, and $50 \mu \mathrm{L}$ of a $0.2 \%$ solution

562 of RapiGest (diluted in $\mathrm{NH}_{4} \mathrm{HCO}_{3}$, Waters, 186001861). Next, samples were heated at $80{ }^{\circ} \mathrm{C}$ for

$56315 \mathrm{~min}$ and cooled to RT before addition of $1 \mu \mathrm{g}$ of trypsin (Promega, V511A). Samples were

564 digested at $37{ }^{\circ} \mathrm{C}$ overnight. To hydrolyze the RapiGest, $20 \mu \mathrm{L}$ of $5 \%$ trifluoroacetic acid (TFA)

565 was added to samples which were incubated at $37^{\circ} \mathrm{C}$ for $90 \mathrm{~min}$ prior centrifugation at $15000 \mathrm{~g}$ for

56625 min at $4{ }^{\circ} \mathrm{C}$. Samples were desalted using C18 OMIX tips (Agilent Technologies, A57003100)

567 according to the manufacturer's instructions and sample volume was decreased to $20 \mu \mathrm{L}$ using a

568 SpeedVac vacuum concentrator. Samples were stored at $4{ }^{\circ} \mathrm{C}$ prior to analysis. 
572 (P6) and pkmt1::tn (P4) and pkmt2::tn (P3) bacteria were centrifuged at 14,000g for 3 min at room

573 temperature. Next, to release the surface protein fraction, the bacterial pellets were resuspended in

574 lysis buffer (50 mM Tris-HCI, $\mathrm{pH}$ 7.5, $150 \mathrm{mM} \mathrm{NaCl}, 1 \mathrm{mM}$ EDTA and 10\% glycerol),

575 supplemented with $0.0031 \% \mathrm{v} / \mathrm{v}$ lysonase (Millipore, 71230), the deubiquitylase inhibitor PR619

576 (Life Sensor, SI9619) at a final concentration of $20 \mu \mathrm{M}$, and $0.8 \% \mathrm{w} / \mathrm{v}$ octyl $\beta$-D-glucopyranoside

577 (Sigma, O8001), and incubated on ice for $10 \mathrm{~min}$ with occasional pipetting of samples to break

578 pellet into smaller pieces. Subsequently, the lysate was cleared by centrifugation at 14,000 $g$ at 4

$579{ }^{\circ} \mathrm{C}$ for $5 \mathrm{~min}$ and incubated with equilibrated agarose TUBE-1 (Life Sensor, UM401) for $3 \mathrm{~h}$, at 4

$580{ }^{\circ} \mathrm{C}$. After binding of polyubiquitylated proteins to TUBE-1, agarose beads were washed 1 time

581 with TBS supplemented with $0.05 \%$ Tween and $5 \mathrm{mM}$ EDTA, and subsequently 3 times with TBS

582 only (no Tween or EDTA) and centrifuged at 5,000g for $5 \mathrm{~min}$. To prepare samples for MS 583 analysis, enriched proteins were digested at $37{ }^{\circ} \mathrm{C}$ overnight on agarose beads in RapiGest SF

584 solution (Waters, 186001861) supplemented with $0.75 \mu \mathrm{g}$ trypsin (Promega, V511A). The reaction

585 was stopped using 1\% TFA (Sigma, T6508). Octyl $\beta$-D-glucopyranoside was extracted using

586 water-saturated ethyl acetate (Sigma, 34858). Prior to submission of samples for mass

587 spectrometry analysis, samples were desalted using C18 OMIX tips (Agilent Technologies, 588 A57003100), according to the manufacturer's instructions.

590 Liquid chromatography-mass spectrometry

591 Samples of proteolytically digested proteins were analyzed using a Synapt G2-Si ion

592 mobility mass spectrometer that was equipped with a nanoelectrospray ionization source (Waters).

593 The mass spectrometer was connected in line with an Acquity M-class ultra-performance liquid 
594 chromatography system that was equipped with trapping (Symmetry C18, inner diameter: $180 \mu \mathrm{m}$,

595 length: $20 \mathrm{~mm}$, particle size: $5 \mu \mathrm{m}$ ) and analytical (HSS T3, inner diameter: $75 \mu \mathrm{m}$, length: 250

$596 \mathrm{~mm}$, particle size: $1.8 \mu \mathrm{m}$ ) columns (Waters). Data-independent, ion mobility-enabled, high-

597 definition mass spectra and tandem mass spectra were acquired in the positive ion mode (23-25).

598 Raw data acquisition was controlled using MassLynx software (version 4.1), and tryptic peptide

599 identification and relative quantification using a label-free approach $(26,27)$ were performed using

600 Progenesis QI for Proteomics software (version 4.0, Waters). Raw data were searched against

601 Rickettsia parkeri and Chlorocebus sabaeus protein databases (National Center for Biotechnology

602 Information, NCBI) to identify tryptic peptides, allowing for up to three missed proteolytic

603 cleavages, with diglycine-modified lysine (i.e., ubiquitylation remnant) and methylated lysine as

604 variable post-translational modifications. Calculation of the percentage of lysine methylation

605 (mono, di, tri or unmethylated), for each bacterial strain, was performed by dividing the abundance

606 of a residue/protein bearing a modification by the total abundance and multiplying by 100 . Data-

607 dependent analysis was performed using an UltiMate3000 RSLCnano liquid chromatography

608 system that was connected in line with an LTQ-Orbitrap-XL mass spectrometer equipped with a

609 nanoelectrospray ionization source, and Xcalibur (version 2.0.7) and Proteome Discoverer

610 (version 1.3, Thermo Fisher Scientific, Waltham, MA) software, as described elsewhere (28).

611

612 Localization of tagged ubiquitin and ubiquitin pull-downs

613 To assess localization of 6xHis-ubiquitin during infection, confluent Vero cells grown in

614 24-well plates with coverslips were transfected with $2 \mu \mathrm{g}$ of pCS2-6xHis-ubiquitin plasmid DNA

615 using Lipofectamine 2000 (Invitrogen, 11668-019) for $6 \mathrm{~h}$ in Opti-MEM (Gibco, 31985-070).

616 Subsequently, media were exchanged to media without transfection reagent, and cells were 
617 incubated overnight at $37^{\circ} \mathrm{C}$ and $5 \% \mathrm{CO}_{2}$. The following day ( $\sim 16 \mathrm{~h}$ after transfection), transfected

618 cells were infected with purified WT or mutant bacteria at a MOI of 1. At 28 h.p.i., infected cells

619 were fixed with 4\% PFA diluted in PBS, pH 7.4 for 10 min, then washed 3 times with PBS. Primary

620 anti-6xHis monoclonal mouse antibody (Clontech, 631212, diluted 1:1,000) was used to detect

621 6xHis-ubiquitin in samples permeabilized with $0.5 \%$ Triton-X100, and a goat anti-mouse Alexa-

622568 (Invitrogen, A11004) to detect the primary 6xHis antibody. Hoechst (Thermo Scientific,

623 62249, diluted 1:2500) was used to detect host and bacterial DNA. Samples were imaged as

624 already described.

625 For ubiquitin pull-downs, confluent Vero cells grown in 6-well plates were transfected and

626 infected as described above. At 28 h.p.i., cells were washed once with 1x PBS, pH 7.4, and

627 subsequently lysed in urea lysis buffer (8 M urea, $50 \mathrm{mM}$ Tris-HCI, pH 8.0, $300 \mathrm{mM}$ NaCI, 50

$628 \mathrm{mM} \mathrm{Na}_{2} \mathrm{HPO}_{4}, 0.5 \%$ Igepal CA-630 (Sigma, I8896)) for $20 \mathrm{~min}$ at RT. Subsequently, samples

629 were sonicated, and lysate was cleared by centrifugation at $15000 \mathrm{~g}$ for $15 \mathrm{~min}$ at room temperature.

630 Prior to incubation with Ni-NTA resin, an aliquot was saved for the input sample. 6xHis-ubiquitin

631 conjugates were purified by incubation and rotation with Ni-NTA resin for $3 \mathrm{~h}$, at RT, in the

632 presence of $10 \mathrm{mM}$ imidazole. Beads were washed 3 times with urea lysis buffer and 1 time with

633 urea lysis buffer lacking Igepal CA-630. Ubiquitin conjugates were eluted at $65{ }^{\circ} \mathrm{C}$ for 15 min in

634 2x Laemmli buffer containing $200 \mathrm{mM}$ imidazole and 5\% 2-mercaptoetanol (Sigma, M6250),

635 vortexed for 90 seconds, and centrifuged at $5000 \mathrm{~g}$ for $5 \mathrm{~min}$ at RT. Eluted and input proteins were

636 detected by SDS-PAGE followed by western blotting, as described above. 
641 Act. The UC Berkeley IACUC is fully accredited by the Association for the Assessment and

642 Accreditation of Laboratory Animal Care International and adheres to the principles of the Guide

643 for the Care and Use of Laboratory Animals. Infections were performed in a biosafety level 2

644 facility. Mice were age-matched between 8 and 18 weeks old. Mice were selected for experiments

645 based on their availability, regardless of sex. All mice were healthy at the time of infection and

646 were housed in microisolator cages and provided chow and water. Littermates of the same sex

647 were randomly assigned to experimental groups. For infections, $R$. parkeri was prepared by

648 diluting 30\%-prep bacteria into cold sterile $1 \mathrm{x}$ PBS to $5 \times 10^{6} \mathrm{PFU}$ per $200 \mathrm{~mL}$. Bacterial

649 suspensions were kept on ice during injections. Mice were exposed to a heat lamp while in their

650 cages for approximately $5 \mathrm{~min}$, and then each mouse was moved to a mouse restrainer (Braintree,

651 TB-150 STD). The tail was sterilized with 70\% ethanol, and $200 \mu \mathrm{L}$ of bacterial suspensions were

652 injected using 30.5-gauge needles into the lateral tail vein. Body temperatures were monitored

653 using a rodent rectal thermometer (BrainTree Scientific, RET-3). Mice were monitored daily for

654 clinical signs of disease, such as hunched posture, lethargy, or scruffed fur. If a mouse displayed

655 severe signs of infection, as defined by a reduction in body temperature below $90{ }^{\circ} \mathrm{F}$ or an inability

656 to move around the cage normally, the animal was immediately and humanely euthanized using

$657 \mathrm{CO}_{2}$ followed by cervical dislocation, according to IACUC-approved procedures (16).

659 Statistical analysis, experimental variability and reproducibility

660 Statistical parameters and significance are reported in the legends. Data were considered 661 to be statistically significant when $\mathrm{p}<0.05$, as determined by a one-way ANOVA with Dunnett's 
662 post-hoc test, a Kruskal-Wallis test with Dunn's post-hoc test, a Brown-Forsyth and Welch 663 ANOVA with Dunnett's post-hoc test, or a two-way ANOVA (all two-sided). Statistical analysis 664 was performed using PRISM 6 software (GraphPad Software). If not otherwise described, $n$ 665 indicates the number of independent biological experiments executed at different times. 